\title{
Multi-wavelength characterization of the blazar S5 0716+714 during an unprecedented outburst phase ${ }^{\star}$
}

MAGIC Collaboration: M. L. Ahnen ${ }^{1}$, S. Ansoldi ${ }^{2,20}$, L. A. Antonelli ${ }^{3}$, C. Arcaro ${ }^{4,27}$, D. Baack ${ }^{5}$, A. Babić ${ }^{6}$, B. Banerjee ${ }^{7}$, P. Bangale ${ }^{8}$,

U. Barres de Almeida ${ }^{8,9}$, J. A. Barrio ${ }^{10}$, J. Becerra González ${ }^{11}$, W. Bednarek ${ }^{12}$, E. Bernardini ${ }^{4,13,23,27}$, R. Ch. Berse ${ }^{5}$, A. Berti ${ }^{2,24}$,

W. Bhattacharyya ${ }^{13}$, A. Biland ${ }^{1}$, O. Blanch ${ }^{14}$, G. Bonnoli ${ }^{15}$, R. Carosi ${ }^{15}$, A. Carosi ${ }^{3}$, G. Ceribella ${ }^{8}$, A. Chatterjee ${ }^{7}$, S. M. Colak $^{14}$, P. Colin $^{8}$, E. Colombo ${ }^{11}$, J. L. Contreras ${ }^{10}$, J. Cortina ${ }^{14}$, S. Covino ${ }^{3}$, P. Cumani ${ }^{14}$, P. Da Vela ${ }^{15}$, F. Dazzi ${ }^{3}$, A. De Angelis ${ }^{4,27}$, B. De Lotto ${ }^{2}$, M. Delfino ${ }^{14,25}$,

J. Delgado ${ }^{14}$, F. Di Pierro ${ }^{4,27}$, A. Domínguez ${ }^{10}$, D. Dominis Prester ${ }^{6}$, D. Dorner ${ }^{16}$, M. Doro ${ }^{4,27}$, S. Einecke ${ }^{5}$, D. Elsaesser ${ }^{5}$,

V. Fallah Ramazani ${ }^{17}$, A. Fernández-Barral ${ }^{4,14,27}$, D. Fidalgo ${ }^{10}$, M. V. Fonseca ${ }^{10}$, L. Font ${ }^{18}$, C. Fruck ${ }^{8}$, D. Galindo ${ }^{19}$, S. Gallozzi $^{3}$, R. J. García López ${ }^{11}$, M. Garczarczyk ${ }^{13}$, M. Gaug ${ }^{18}$, P. Giammaria ${ }^{3}$, N. Godinović ${ }^{6}$, D. Gora ${ }^{13}$, D. Guberman ${ }^{14}$, D. Hadasch $^{20}$, A. Hahn $^{8}$, T. Hassan ${ }^{14}$, M. Hayashida ${ }^{20}$, J. Herrera ${ }^{11}$, J. Hose ${ }^{8}$, D. Hrupec ${ }^{6}$, K. Ishio ${ }^{8}$, Y. Konno ${ }^{20}$, H. Kubo ${ }^{20}$, J. Kushida ${ }^{20}$, D. Kuveždić ${ }^{6}$, D. Lelas ${ }^{6}$, E. Lindfors ${ }^{17, \star \star}$, S. Lombardi ${ }^{3}$, F. Longo ${ }^{2,24}$, M. López ${ }^{10}$, C. Maggio ${ }^{18}$, P. Majumdar ${ }^{7}$, M. Makariev ${ }^{21}$, G. Maneva ${ }^{21}$, M. Manganaro ${ }^{11, \star \star}$, K. Mannheim ${ }^{16}$, L. Maraschi ${ }^{3}$, M. Mariotti ${ }^{4,27}$, M. Martínez ${ }^{14}$, S. Masuda ${ }^{20}$, D. Mazin ${ }^{8,20}$, K. Mielke ${ }^{5}$ M. Minev ${ }^{21}$, J. M. Miranda $^{15}$, R. Mirzoyan ${ }^{8}$, A. Moralejo ${ }^{14}$, V. Moreno ${ }^{18}$, E. Moretti ${ }^{8}$, T. Nagayoshi ${ }^{20}$, V. Neustroev ${ }^{17}$, A. Niedzwiecki ${ }^{12}$, M. Nievas Rosillo $^{10}$, C. Nigro $^{13}$, K. Nilsson ${ }^{17}$, D. Ninci ${ }^{14}$, K. Nishijima ${ }^{20}$, K. Noda ${ }^{14}$, L. Nogués ${ }^{14}$, S. Paiano ${ }^{4,27}$, J. Palacio ${ }^{14}$, D. Paneque ${ }^{8}$, R. Paoletti ${ }^{15}$, J. M. Paredes ${ }^{19}$, G. Pedaletti ${ }^{13, \star \star}$, M. Peresano ${ }^{2}$, M. Persic ${ }^{2,26}$, P. G. Prada Moroni ${ }^{22}$, E. Prandini ${ }^{4,27}$, I. Puljak ${ }^{6}$, J. R. Garcia ${ }^{8}$, I. Reichardt ${ }^{4,27}$, W. Rhode ${ }^{5}$, M. Ribó ${ }^{19}$, J. Rico ${ }^{14}$, C. Righi ${ }^{3}$, A. Rugliancich ${ }^{15}$, T. Saito ${ }^{20}$, K. Satalecka ${ }^{13}$, T. Schweizer ${ }^{8}$, J. Sitarek ${ }^{12,20}$, I. Šnidarić ${ }^{6}$, D. Sobczynska $^{12}$,

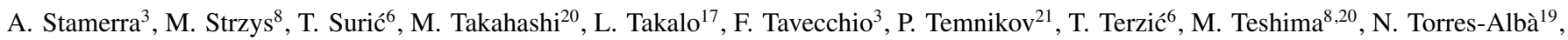

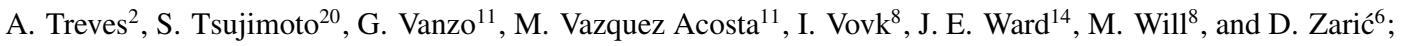

Fermi-LAT Collaboration: D. Bastieri ${ }^{27}$, D. Gasparrini ${ }^{28}$, B. Lott $^{29}$, B. Rani ${ }^{30, \star \star}$, D. J. Thompson ${ }^{30}$;

MWL Collaborators: I. Agudo ${ }^{31}$, E. Angelakis ${ }^{32}$, G. A. Borman ${ }^{33}$, C. Casadio ${ }^{32,31}$, T. S. Grishina ${ }^{34}$, M. Gurwell ${ }^{35}$, T. Hovatta ${ }^{36,37,38}$, R. Itoh ${ }^{39}$,

E. Järvelä ${ }^{36,37}$, H. Jermak ${ }^{47}$, S. Jorstad ${ }^{34,40}$, E. N. Kopatskaya ${ }^{34}$, A. Kraus ${ }^{32}$, T. P. Krichbaum ${ }^{32}$, N. P. M. Kuin ${ }^{41}$, A. Lähteenmäki ${ }^{36,37}$,

V. M. Larionov ${ }^{34,42}$, L. V. Larionova ${ }^{34}$, A. Y. Lien $^{30}$, G. Madejski ${ }^{43}$, A. Marscher ${ }^{40}$, I. Myserlis ${ }^{32}$, W. Max-Moerbeck ${ }^{32}$, S. N. Molina ${ }^{31}$, D. A. Morozova ${ }^{34}$, K. Nalewajko ${ }^{44}$, T. J. Pearson ${ }^{45}$, V. Ramakrishnan ${ }^{36}$, A. C. S. Readhead ${ }^{45}$, R. A. Reeves ${ }^{46}$, S. S. Savchenko ${ }^{34}$, I. A. Steele ${ }^{47}$, M. Tornikoski ${ }^{36}$, Yu. V. Troitskaya ${ }^{34}$, I. Troitsky ${ }^{34}$, A. A. Vasilyev ${ }^{34}$, J. Anton Zensus ${ }^{32}$

(Affiliations can be found after the references)

Received 20 January 2018 / Accepted 30 June 2018

\section{ABSTRACT}

Context. The BL Lac object S5 0716+714, a highly variable blazar, underwent an impressive outburst in January 2015 (Phase A), followed by minor activity in February (Phase B). The MAGIC observations were triggered by the optical flux observed in Phase A, corresponding to the brightest ever reported state of the source in the $R$-band.

Aims.The comprehensive dataset collected is investigated in order to shed light on the mechanism of the broadband emission.

Methods. Multi-wavelength light curves have been studied together with the broadband spectral energy distributions (SEDs). The sample includes data from Effelsberg, OVRO, Metsähovi, VLBI, CARMA, IRAM, SMA, Swift-UVOT, KVA, Tuorla, Steward, RINGO3, KANATA, AZT-8+ST7, Perkins, LX-200, Swift-XRT, NuSTAR, Fermi-LAT and MAGIC.

Results. The flaring state of Phase A was detected in all the energy bands, providing for the first time a multi-wavelength sample of simultaneous data from the radio band to the very-high-energy (VHE, $E>100 \mathrm{GeV}$ ). In the constructed SED, the Swift-XRT+NuSTAR data constrain the transition between the synchrotron and inverse Compton components very accurately, while the second peak is constrained from $0.1 \mathrm{GeV}$ to $600 \mathrm{GeV}$ by Fermi+MAGIC data. The broadband SED cannot be described with a one-zone synchrotron self-Compton model as it severely underestimates the optical flux in order to reproduce the X-ray to $\gamma$-ray data. Instead we use a two-zone model. The electric vector position angle (EVPA) shows an unprecedented fast rotation. An estimation of the redshift of the source by combined high-energy $(\mathrm{HE}, 0.1 \mathrm{GeV}<E<100 \mathrm{GeV})$ and VHE data provides a value of $z=0.31 \pm 0.02_{\text {stats }} \pm 0.05_{\text {sys }}$, confirming the literature value.

Conclusions. The data show the VHE emission originating in the entrance and exit of a superluminal knot in and out of a recollimation shock in the inner jet. A shock-shock interaction in the jet seems responsible for the observed flares and EVPA swing. This scenario is also consistent with the SED modeling.

Key words. BL Lacertae objects: individual: S5 0716+714 - galaxies: active - galaxies: jets - gamma rays: galaxies

\footnotetext{
* The complete data set shown in Figs. 1 and 2, the data points shown in Figs. 8 and 9 and Table A.1 are only available at the CDS via anonymous ftp to cdsarc.u-strasbg.fr $(130.79 .128 .5)$ or via http://cdsarc.u-strasbg.fr/viz-bin/qcat?J/A+A/619/A45

$\star \star$ Corresponding authors: M. Manganaro, email: manganaro@iac.es; B. Rani, NPP fellow, email: bindu.rani@nasa.gov; E. Lindfors, email: elilin@utu.fi
} 


\section{Introduction}

The blazar S5 $0716+714$ is a BL Lac object characterized by extreme variability in almost all energy bands. Because of the featureless optical continuum (Paiano et al. 2017) it is hard to estimate its redshift. Nilsson et al. (2008) claim a value of $z=0.31 \pm 0.08$ based on the photometric detection of the host galaxy. Detection of intervening Ly $\alpha$ systems in the ultraviolet (UV) spectrum of the source confirms the earlier estimates with a redshift value $z<0.32$ (95\% confidence; Danforth et al. 2013).

The familiar shape of the spectral energy distribution (SED) of blazars, in which the two bumps are identified as synchrotron and inverse Compton (IC), respectively, is used for their classification. In the case of S5 0716+714, the first peak of the SED is located between $10^{14}$ and $10^{15} \mathrm{~Hz}$, leading to a classification as an intermediate synchrotron peaked blazar, also referred to as an intermediate-peaked BL Lac object (IBL; Giommi et al. 1999; Ackermann et al. 2011).

Owing to its remarkable variability, S5 $0716+714$ has been the subject of many optical monitoring campaigns (Wagner et al. 1996; Montagni et al. 2006; Rani et al. 2011,2013,2015). Several authors carried out flux variability studies (e.g., Quirrenbach et al. 1991; Wagner et al. 1996) and morphological/kinematic studies at radio frequencies (Antonucci et al. 1986; Witzel et al. 1988; Jorstad et al. 2001; Bach et al. 2005; Rastorgueva et al. 2011). The observed intraday variability at radio wavelengths is likely to be a mixture of intrinsic and external (due to interstellar scintillation) mechanisms. Wagner et al. (1996) reported a significant correlation between optical-radio flux variations at dayto-day timescales. Rani et al. (2010) reported the detection of $\sim 15$-min quasi-periodic oscillations (QPOs) at optical frequencies, the shortest observed in any blazar so far.

The study of the broadband flux variability is a difficult task because of the complexity of the flaring activity, which can vary rapidly on a timescale of a few hours to days, while on a timescale of $\sim 1 \mathrm{yr}$, a broader and slower variability trend has been reported (Raiteri et al. 2003; Rani et al. 2013). As seen by Very Long Baseline Interferometry (VLBI) studies (Bach et al. 2005; Britzen et al. 2009), the source presents a core-dominated jet pointing towards the north. Very Large Array observations show a halo-like jet misaligned with it by $\sim 90^{\circ}$ on kiloparsec scales (Antonucci et al. 1986). Britzen et al. (2009) suggest that there is an apparent stationarity of jet components relative to the core. However, recently apparent speeds of $\sim 40 \mathrm{c}$ have been reported in Rastorgueva et al. (2011), Larionov et al. (2013), Lister et al. (2013) and Rani et al. (2015). Britzen et al. (2009), Rastorgueva et al. (2011) and Rani et al. (2014) observed nonradial motion and wiggling component trajectories in the inner milliarcsecond jet region.

Observations by BeppoSAX (Tagliaferri et al. 2003) and $X M M-N e w t o n$ (Foschini et al. 2006) provide evidence for a concave X-ray spectrum in the $0.1-10 \mathrm{keV}$ band, a signature of the presence of both the steep tail of the synchrotron emission and the rising part of IC spectrum.

The Energetic Gamma Ray Experiment Telescope (EGRET) on board the Compton Gamma-ray Observatory (CGRO) detected high-energy ( $\mathrm{HE}, 0.1 \mathrm{GeV}<E<100 \mathrm{GeV}$ ) $\gamma$-ray emission from S5 0716+714 several times from 1991 to 1996 (Lin et al. 1995; Hartman et al. 1999). Two strong $\gamma$-ray flares in September and October 2007 were detected in the source with AGILE (Astro-rivelatore Gamma a Immagini LEggero, Chen et al. 2008) in the HE range. These authors also carried out SED modeling of the source with two synchrotron self-Compton (SSC) emitting components, representative of slowly- and rapidly variable components, respectively.

The source S50716+714 was first detected in the veryhigh-energy (VHE, $E>100 \mathrm{GeV}$ ) range by MAGIC (Major Atmospheric Gamma-ray Imaging Cherenkov telescopes) with $5.8 \sigma$ significance level in November 2007 and then in April 2008 (Anderhub et al. 2009) during an optical flare. At that time, MAGIC was working with a single telescope and the energy threshold of the telescope for such a high zenith range $\left(47^{\circ}<z d<55^{\circ}\right)$ was $400 \mathrm{GeV}$. The analysis of multi-wavelength data suggested a correlation between the VHE $\gamma$-ray and optical emission. A structured jet model, composed of a fast spine surrounded by a slower-moving layer (Ghisellini et al. 2005; Tavecchio \& Ghisellini 2009) better described the data compared to a simple one-zone SSC model. This source is also among the bright blazars in the Fermi-LAT (Large Area Telescope) bright active galactic nuclei (AGNs) sample (LBAS; Abdo et al. 2010a) and in the Fermi third catalog (Acero et al. 2015 ) it is one of the blazars with the highest variability index. The combined GeV-TeV spectrum of the source might display an absorption-like feature in the $10-100 \mathrm{GeV}$ energy range (Senturk et al. 2013).

Apart from the $\gamma$-ray/optical connection, which is to be expected in one-zone, single-population leptonic models, another interesting feature of the broadband activity was reported in Rani et al. (2013), in which the authors found $\gamma$-ray/optical flux variations preceding the radio variability by $\sim 65$ days. An orphan X-ray flare was detected in 2009 by Swift-XRT but no VHE observations were available to check if there was a counterpart in the VHE range. The behavior of the source in the past years can be contextualized in the scenario of a shockwave propagating along a helical path in the blazar's jet (Marscher et al. 2008; Larionov et al. 2013).

Recently, more attention was given to the electric vector position angle (EVPA) swings: Rani et al. (2014) found a significant correlation between the inner jet outflow orientation and $\gamma$-ray flux variations, showing how the morphology of the inner jet has a strong connection with the $\gamma$-ray flares. Chandra et al. (2015a) studied the rapid variation in the degree of polarization (PD) and in the polarization angle (PA) within the helical magnetic field model (HMFM; Zhang et al. 2014), explaining such features as most likely due to reconnections in the emission region of the jet.

In the present work, we report the results of a multiwavelength (MWL) campaign organized to follow an unprecedented outburst phase of the blazar S50716+714 during January 2015. The source was detected at its highest brightness so far at optical and infrared (IR) bands. On January 11, 2015, (MJD 57033) the near-infrared (NIR) photometry reported an increase of its flux by a factor of 2.5 in the NIR band in a rather short lapse of 12 days (Carrasco et al. 2015; Chandra et al. 2015b). During the night of 18 January 2015 (MJD 57040), the source was detected at its highest brightness so far, with $R$ band magnitude 11.71 (Bachev \& Strigachev 2015). The TeV observations triggered to follow the exceptionally high optical state detected the source at energies above $150 \mathrm{GeV}$ (Mirzoyan 2015) and went on until the flaring activity faded away.

The paper is structured as follows. In Sect. 2, we describe the various instruments involved in the observations and the analysis of their data. In Sect. 3, we present the multi-wavelength light curves and discuss the peculiarities of the source activity in the different bands. Section 4 is devoted to the jet analysis of the object by VLBI. In Sect. 5, the spectral fitting of $N u S$ $T A R$ and Swift-XRT data is shown. In Sect. 6, the VHE spectra 
obtained by MAGIC and the redshift estimation using MAGIC and Fermi-LAT simultaneous data are presented, and in Sect. 7 the broadband SED for the two phases considered is discussed together with the modeling of the source. Results and Conclusions are given in Sect. 8.

\section{Observations and analysis}

\subsection{VHE $\gamma$-ray observations}

MAGIC is a stereoscopic system consisting of two Imaging Atmospheric Cherenkov Telescopes, each $17 \mathrm{~m}$ in diameter, located at the Observatorio del Roque de los Muchachos, on the Canary Island of La Palma. The current sensitivity for mediumzenith observations $\left(30^{\circ}<z d<45^{\circ}\right)$ above $210 \mathrm{GeV}$ is $0.76 \pm$ $0.04 \%$ of the Crab Nebula's flux in 50 h (Aleksić et al. 2016). On 19 January 2015 (MJD 57041), triggered by the high optical state and by high-energy photons detected by Fermi-LAT, MAGIC started to observe S5 0716+714. On 23 January 2015 (MJD 57045), the significance of the signal was found to be $6.4 \sigma$, and it reached a maximum value of $13.2 \sigma$ on 26 January (MJD 57048): the flux increased from $(4.1 \pm 1.1) \times 10^{-11} \mathrm{~cm}^{-2} \mathrm{~s}^{-1}$ to $(8.9 \pm 1.1) \times 10^{-11} \mathrm{~cm}^{-2} \mathrm{~s}^{-1}$ above $150 \mathrm{GeV}$, which is the highest level ever detected in the VHE band for this source. The subsequent activity of S5 $0716+714$ in the VHE range was detected by MAGIC on 13 February (MJD 57066), this time lasting four days only, up to the 16 February (MJD 57069). In the present work, the two multi-wavelength periods of observations that include MAGIC data are indicated as Phase A, from 18 to 27 January 2015 (MJD 57040 to MJD 57050), and Phase B, from 12 to 17 February 2015 (MJD 57065 to MJD 57070). We collected $17.74 \mathrm{~h}$ of data in the zenith angle range of $40^{\circ}<z d<50^{\circ}$ and the analysis was performed using the standard MAGIC analysis framework MARS (Zanin et al. 2013; Aleksić et al. 2016). After the applied quality cuts, the surviving events amount to $17.46 \mathrm{~h}$ in total. A statistical significance of $18.9 \sigma$ was found for the full sample after cuts. The significance of the signal was calculated as in Eq. (17) in Li \& Ma (1983). For the MAGIC SED, the systematic uncertainty on the flux normalization was estimated to be $11 \%$, and on the spectral slope \pm 0.15 . The analysis energy threshold is $\sim 125 \mathrm{GeV}$, measured as the peak of the Monte Carlo (MC) energy distribution for a source with the spectral shape of S50716+714. A full description of the MAGIC systematic uncertainties can be found in Aleksić et al. (2016).

\subsection{HE $\gamma$-ray observations}

The HE $\gamma$-ray $(0.1-300 \mathrm{GeV})$ observations for a time period between 1 November 2014 (MJD 56962) and 31 July 2015 (MJD 57234) were obtained in a survey mode by the Fermi-LAT (Atwood et al. 2009). The LAT data were analyzed using the standard ScienceTools ${ }^{1}$ (software version v10.01.01, pass8) with instrument response function P8R2_SOURCE_V6. Photons in the source event class were selected for the analysis. We analyzed a region of interest $(\mathrm{ROI})$ of $10^{\circ}$ in radius centered at the position of S50716+714 using a maximum-likelihood algorithm (Mattox et al. 1996). We included all 54 sources of the 3FGL catalog (Acero et al. 2015) within $20^{\circ}$ of the position of S5 0716+714 in the unbinned likelihood analysis. Model parameters for sources within $5^{\circ}$ of the ROI are kept free; we kept the model parameters for the rest fixed to their catalog values.

https://fermi.gsfc.nasa.gov/ssc/data/analysis/
To investigate the source variability at $E>100 \mathrm{MeV}$, we generated the daily binned photon flux and index curves using unbinned likelihood analysis. The daily binned data were computed by modeling the spectra by a power-law model (PWL, $N(E)=N_{0} E^{-\Gamma}, N_{0}$ : prefactor, and $\Gamma$ : power law index). We examined the spectral behavior over the whole energy range with a PWL model fitting over equally spaced logarithmic energy bins with $\Gamma$ kept constant and equal to the value fit over the whole range. Even if in the Fermi third catalog (Acero et al. 2015) the source spectrum is described by a log parabola model, for the present dedicated analysis the PWL model better fits the data. The upper limits for the light curve are shown as gray triangles in the second panel from top in Fig. 1 and they were calculated for test statistics $<9$.

\subsection{X-ray and optical/UV observations}

\subsubsection{NUSTAR}

The exceptional flare from the object triggered a Target of Opportunity observation of the object by NUSTAR. NUSTAR, a NASA Small Explorer satellite sensitive in the hard X-ray band, features two multilayer-coated telescopes, focusing the reflected $\mathrm{X}$-rays on the pixellated CdZnTe focal plane modules with the half-power diameter of an image of a point source of $\sim 1^{\prime}$. It provides a bandpass of $3-79 \mathrm{keV}$ with a spectral resolution of $\sim 1 \mathrm{keV}$. For more details, see Harrison et al. (2013).

After screening for the South Atlantic Anomaly passages and Earth occultation, the pointing (with OBSID 90002003002) resulted in roughly $14.9 \mathrm{ks}$ of net observing time on 24 January 2015 (MJD 57046). After processing the raw data with the NUSTAR Data Analysis Software (NuSTARDAS) package v.1.3.1 (via the script nupipeline), the source data were extracted from a region of $45^{\prime \prime}$ radius, centered on the centroid of $\mathrm{X}$-ray emission, while the background was extracted from a $1.5^{\prime}$ radius region roughly $5^{\prime}$ southwest of the source location. Spectra were binned in order to have at least 30 counts per rebinned channel. We considered the spectral channels corresponding nominally to the $3-60 \mathrm{keV}$ energy range, where the source was robustly detected. The mean net (background-subtracted) count rates were $0.174 \pm 0.003$ and $0.165 \pm 0.003 \mathrm{cts} \mathrm{s}^{-1}$, respectively, for the modules FPMA and FPMB. We found no variability of the source as a function of time within the NUSTAR observation, and we summed the data into one spectral file for each focal plane module.

\subsubsection{Swift-XRT}

The multi-epochs (35) event list obtained by the X-ray Telescope (XRT; Burrows et al. 2004), onboard the Neil Gehrels Swift satellite in the period 1 January 2015 (MJD 57023.2) to 28 February 2015 (MJD 57081.2) with a total exposure time of $\sim 16.81 \mathrm{~h}$ was downloaded from publicly available SWIFTXRLOG (SwiftXRT Instrument Log). This was processed using the procedure described in Fallah Ramazani et al. (2017). All these observations have been performed in photon counting (PC) mode, with an average integration time of $1.7 \mathrm{ks}$ each. The equivalent Galactic hydrogen column density is fixed to the value of $n_{\mathrm{H}}=3.11 \times 10^{20} \mathrm{~cm}^{-2}$ (Kalberla et al. 2005). We performed spectral fits to all 35 epochs using a simple power law model with Galactic absorption. This model provides a good fit, and the $0.3-10 \mathrm{keV}$ spectral index is $2.72 \pm 0.1$. We discuss the Swift observations more extensively in Sect. 3. We note specifically that one of the pointings was simultaneous with the NUSTAR observation (at MJD 57045 - 24 January 
2015) and we use that observation for joint XRT-NuSTAR spectral fitting in Sect. 5.

\subsubsection{Swift-UVOT}

Photometric observations by the Swift Ultra-Violet and Optical Telescope (UVOT) instrument were made in the three UV (uvw2, uvm2, and uvw1) and three optical $(u, b$, and $v$ ) filters in both imaging and event mode. During the February 2015 observations, good coverage in the UV and $u$ bands exist and in particular uvm 2 data in event mode were obtained in order to resolve short timescale variability in the UV.

The UVOT data reduction used the Heasarc Heasoft version 6.16 and Swift CALDB (September 2013). The event mode data were typically $800 \mathrm{~s}$ exposures and were binned into shorter time slices, converted into images, and then aspect corrected.

This analysis allowed for the identification of a few observations for which the pointing drift was too large, and those were excluded from further analysis. Data taken in image mode were similarly validated. The magnitudes were determined from the images using the UVOTMAGHIST program using the standard calibration (Poole et al. 2008; Breeveld et al. 2011). The details of the event mode data processing are as follows: GTI extensions were created in the event file for the desired time intervals; UVOTATTJUMPCORR was run to improve the attitude file; and COORDINATOR and UVOTSCREEN were used to correct the event file, which was then processed using UVOTIMAGE so that the individual data could be inspected.

\subsection{Optical observations and polarimetry data}

The Tuorla Blazar monitoring program ${ }^{2}$ collects blazar optical light curves in the $R$ band from several observatories. The present work shows, in particular, data from the $1.03 \mathrm{~m}$ telescope at Tuorla Observatory, Finland, and the $35-\mathrm{cm}$ telescope at the KVA observatory on La Palma, Canary Islands, Spain. The data are analyzed with a semi-automatic pipeline using standard procedures (Nilsson et al. 2018)

The Boston University (BU) group uses the 1.83-m Perkins Telescope at Lowell Observatory (Flagstaff, AZ) to carry out optical observations of a sample of $\gamma$-ray blazars, including S5 0716+714. The telescope is equipped with the PRISM camera operating in photometric (UBVRI) and polarimetric modes. The details of the observations and data reduction can be found in Jorstad et al. (2010).

The optical polarimetric data presented here are complemented by data from the RINGO3 polarimeter on the $2.0 \mathrm{~m}$ fully robotic Liverpool Telescope on the Roque the los Muchachos Observatory (La Palma, Canary Islands, Spain). The RINGO3 data presented here were obtained as part of a monitoring program of bright optical blazars (Jermak et al. 2016). S5 0716+714 is one of the targets regularly monitored by this program, with a time cadence of $\sim 3$ days. The RINGO3 polarimeter acquires polarimetric measurements in three different passbands recorded in the so called "Red", "Green", and "Blue" cameras". Here we only present the data from the "Green" camera (the one with the closest wavelength passband to $R$-band). The RINGO3 data were reduced following the procedure explained in Steele et al. (2017).

$R$-band photometry and polarimetry observations of S50716+714 were performed using the HONIR (Hiroshima

\footnotetext{
2 http://users.utu.fi/kani/1m

3 See the RINGO3 specifications at:

http://telescope.livjm.ac.uk/TelInst/Inst/RING03/
}

Optical and Near-InfraRed camera) instrument installed on the 1.5-m Kanata telescope located at the Higashi-Hiroshima Observatory, Japan (Akitaya et al. 2014). A sequence of photopolarimetric observations consisted of successive exposures at four position angles of a half-wave plate: $0,45,22.5$ and $67.5 \mathrm{deg}$. The data were reduced under the standard procedure of CCD photometry. The aperture photometry was performed using the APPHOT package in $\mathrm{PYRAF}^{4}$, and the differential photometry with a comparison star taken in the same frame of S5 0716+714. The comparison star is located at $\mathrm{RA}=07: 21: 53.44$ and Dec $=+71: 20: 36.0$ (J2000), and its magnitude is $R=14.032$ (UCAC-4 Catalog). The polarization angle is defined as usual (measured from north to east), based on calibrations with polarized stars, HD 183143 and HD 204827 (Schulz \& Lenzen 1983). The systematic error caused by instrumental polarization was smaller than 2 deg using the polarized stars.

Optical photometric and polarimetric data provided by St. Petersburg University are from the 70-cm AZT- 8 telescope of the Crimean Astrophysical Observatory ${ }^{5}$ and the $40 \mathrm{~cm}$ telescope LX-200 in St. Petersburg, both equipped with nearly identical imaging photometers-polarimeters. Polarimetric observations were performed using two Savart plates rotated by $45^{\circ}$ relative to each other (see Larionov et al. 2008b). Instrumental polarization was found via stars located near the object under the assumption that their radiation is unpolarized. This is indicated also by the low level of extinction in the direction of S50716+714 $\left(A_{\mathrm{V}}=0.085 \mathrm{mag} ; A_{\mathrm{R}}=0.067 \mathrm{mag}\right.$; Schlafly \& Finkbeiner 2011).

\subsection{Radio observations}

The $230 \mathrm{GHz}(1.33 \mathrm{~mm})$ flux density data were obtained at the Submillimeter Array (SMA) near the summit of Mauna Kea (Hawaii). S50716+714 is included in an ongoing monitoring program at the SMA to determine the flux densities of compact extragalactic radio sources that can be used as complex gain calibrators at millimeter wavelengths (Gurwell et al. 2007). Potential calibrators are from time to time observed for 3-5 min. Data from this program are updated regularly and are available at the SMA website ${ }^{6}$, while the present analysis was a dedicated one.

The IRAM (Institute for Radio Astronomy in the Millimeter Range) 30-m millimeter radio telescope provided $230 \mathrm{GHz}$ $(1.3 \mathrm{~mm})$ and $86 \mathrm{GHz}(3.5 \mathrm{~mm})$ data that were obtained as part of the POLAMI ${ }^{7}$ (Polarimetric AGN Monitoring at Millimeter Wavelengths) program; see Agudo et al. (2018a, b) and Thum et al. (2018). The POLAMI data of S5 0716+714 were acquired and reduced as described in detail in Agudo et al. (2018a). The CARMA (Combined Array for Research in Millimeterwave Astronomy) data were taken with the eight 3.5-m antennas as part of the Monitoring of $\gamma$-ray AGNs with Radio, Millimeter and Optical Telescopes (MARMOT) project ${ }^{8}$. We used $7.5 \mathrm{GHz}$ of bandwidth with a center frequency of $94 \mathrm{GHz}$. The integration time on S5 0716+714 was 5 min for each observation, which yields a typical root mean square (rms) of 10-110 mJy. Absolute flux density calibration was done using nearby observations of Mars. The observational errors are dominated by the abso-

\footnotetext{
4 http://www.stsci.edu/institute/software_hardware/ pyraf/

5 http://craocrimea.ru/ru

6 http://sma1.sma.hawaii.edu/callist/callist.html

7 http://polami.iaa.es

8 http://www.astro.caltech.edu/marmot/
} 
lute calibration uncertainty, assumed to be $10 \%$. All data were processed using the Multichannel Image Reconstruction Image Analysis and Display (MIRIAD; Sault at al. 1995).

We analyzed Very Long Baseline Array (VLBA) data obtained for S5 0716+714, within the VLBA-BU-Blazar program ${ }^{9}$, which are contemporaneous with the high-energy event in January and February 2015. The data include total and polarized intensity images at $43 \mathrm{GHz}$ at nine epochs from November 2014 to August 2015. They were reduced using the Astronomical Image Processing System (AIPS) ${ }^{10}$ and Difmap ${ }^{11}$ software packages, in the general manner described by Jorstad et al. $(2017,2005)$. The total intensity images were modeled by components with circular Gaussian brightness distributions. This allows us to determine the minimum number of components needed to provide the best fit between the data and model at each epoch, as well as the following parameters of components: flux density, $S$, distance from the core, $r$, position angle with respect to the core, $\Theta$, and size of the component, $a$ (full width at half maximum - FWHM - of the Gaussian). These parameters are given in Table A.1.

The $37 \mathrm{GHz}$ observations were made with the Metsähovi radio telescope. The measurements were made with a $1 \mathrm{GHz}-$ band dual beam receiver centered at $36.8 \mathrm{GHz}$. The observations are $\mathrm{ON}-\mathrm{ON}$ observations, alternating the source and the sky in each feed horn. A typical integration time to obtain one flux density data point is between 1200 and 1800 s. Data points with a signal-to-noise ratio $(S / N)<4$ are handled as non-detections. The flux density scale is set by observations of DR 21 . A detailed description of the data reduction and analysis is given in Teräsranta et al. (1998). The error estimate in the flux density includes the contribution from the measurement rms and the uncertainty of the absolute calibration.

$\mathrm{S} 50716+714$ was observed at $15 \mathrm{GHz}$ as part of a highcadence $\gamma$-ray blazar monitoring program using the Owens Valley Radio Observatory (OVRO) $40 \mathrm{~m}$ telescope (Richards et al. 2011). The OVRO $40 \mathrm{~m}$ uses off-axis dual-beam optics and a cryogenic pseudo-correlation receiver with a $15.0 \mathrm{GHz}$ center frequency and $3 \mathrm{GHz}$ bandwidth. The source is alternated between the two beams in an ON-ON fashion to remove atmospheric and ground contamination. The fast gain variations are corrected using a 180-degree phase switch. Calibration is achieved using a temperature-stable diode noise source to remove receiver gain drifts, and the flux density scale is derived assuming the value of 3.44 Jy at $15.0 \mathrm{GHz}$ in Baars et al. (1997). The systematic uncertainty of about $5 \%$ in the flux density scale is not included in the error bars. Complete details of the reduction and calibration procedure are found in Richards et al. (2011).

Observations at 2.6, 4.8,10, and $15 \mathrm{GHz}$ radio bands were conducted using the Effelsberg $100 \mathrm{~m}$ radio telescope ${ }^{12}$. Measurements for the target source and for the calibrator sources were made quasi-simultaneously using the cross-scan method slewing over the source position, in azimuth and elevation direction in order to gain the desired sensitivity. Subsequently, atmospheric opacity correction, pointing off-set correction, gain correction, and sensitivity correction were applied to the data. For details of the observations and data reduction, we refer to Angelakis et al. (2015).

\footnotetext{
9 www.bu.edu/blazars/VLBAproject.html

10 http://www . aips.nrao. edu/index. shtml

$11 \mathrm{ftp}: / /$ ftp.eso.org/scisoft/scisoft4/sources/difmap/ difmap.html

12 http: //www . mpifr-bonn.mpg.de/en/effelsberg
}

The sources 3C 286, NGC 7027, and 3C 84 have been used as common calibrators for the instruments listed in this section.

\section{Multi-wavelength light curves}

In Fig. 1, we present the multi-wavelength data collected during the course of the campaign. A summary of the most important dates can be found in Table 1 .

The top panel shows the daily-binned MAGIC light curve: in the VHE band, the no-variability hypothesis has been discarded, since the fit for a constant flux resulted in a $\chi^{2} /$ n.d.f. $=42 / 7$ for Phase A and $\chi^{2} /$ n.d.f. $=10 / 3$ for Phase B data. A Gaussian function better fits the flare shape, providing $\chi^{2} /$ n.d.f. $=15 / 5$ and $\chi^{2} /$ n.d.f. $=1.8 / 1$ for the two phases, respectively. Phase A peaks on 25 January, MJD $57047.3 \pm 0.4$ (in Fig. 1 corresponding to the $\mathrm{P} 2$ vertical dashed line) with a flux of $(5.9 \pm 0.5) \times 10^{-11} \mathrm{~cm}^{-2} \mathrm{~s}^{-1}$; the standard deviation of the fit is $\sigma=(2.8 \pm 0.5)$ days. Phase B peaks on 14 February 2015 (MJD 57067.9 \pm 0.2 ) with a corresponding flux of $(5.3 \pm 0.7) \times 10^{-11} \mathrm{~cm}^{-2} \mathrm{~s}^{-1}$ indicated by the vertical dashed line P4. The standard deviation of the fit is $\sigma=(1.2 \pm 0.3)$ days. Intra-day variability (on shorter timescales with respect to the daily binned light curves) was not detected with MAGIC: the light curve was fit at different time intervals down to 5 min, but a constant fit was found to be consistent with the data up to the daily scale, where variability is significant.

The Fermi-LAT daily binned light curve is shown in the second panel from the top: the first peak visible in the curve, marked with the vertical dashed line P1, is the precursor of the whole flaring activity. After P1, (MJD 57038.5, 16 January 2017), which triggered VHE observations, two other peaks are visible in Phase $\mathrm{A}$, and the maximum flux reached in $\mathrm{HE}$ is $(8.8 \pm 1.4) \times 10^{-7} \mathrm{~cm}^{-2} \mathrm{~s}^{-1}$, four times the average flux in $\mathrm{HE}$ for the source from the Fermi-LAT 3FGL catalog (Acero et al. 2015). The photon index for Fermi-LAT observations stays very close to the average Fermi-LAT index of the source, indicated in the corresponding panel of Fig. 1 with the horizontal dashed blue line.

The average integral photon X-ray flux $(0.3-10 \mathrm{keV})$ reported by Swift-XRT in Fig. 1 (fourth panel from top) is $(1.87 \pm 0.46) \times 10^{-11} \mathrm{erg} \mathrm{cm}^{-2} \mathrm{~s}^{-1}$. The $\mathrm{X}$-ray flux peaking at MJD57047 (25 January 2015) with $F_{(0.3-10 \mathrm{keV})}=$ $(3.95 \pm 0.12) \times 10^{-11} \mathrm{erg} \mathrm{cm}^{-2} \mathrm{~s}^{-1}$ which is a factor of approximately two higher than the average flux of the analyzed period. The X-ray spectral index during the analyzed period varies in the range $2.03 \leq \Gamma_{X} \leq 2.56$. The softest spectral index was obtained on the night of the highest X-ray and VHE $\gamma$-ray flux (MJD 57047 - 25 January 2015). The X-ray spectra begin hardening smoothly afterwards for the following 14 consecutive nights. The X-ray spectra on the nights of VHE $\gamma$-ray flares of Phase A and B can be well described by a power law with spectral index of $\Gamma_{\mathrm{X}, \mathrm{MJD}} 57047=2.56 \pm 0.06$ (reduced $\chi^{2} /$ n.d.f. $\left.=0.738 / 29\right)$ and $\Gamma_{\mathrm{X}, \mathrm{MJD} 57067}=2.33 \pm 0.06$ (reduced $\chi^{2} /$ n.d.f. $\left.=0.871 / 26\right)$, respectively.

From the Tuorla optical monitoring, on 18 January 2015 (MJD 57040) the magnitude in the $R$-band reached a value of $\sim 11.5$, higher than the magnitude value of the source in the quiescent state $\left(R_{\mathrm{mag}} \sim 13.5\right)$. Later, the source faded down to $R_{\text {mag }}=12.18 \pm 0.03$ on 20 January 2015 (MJD 57042), flared up again to $R_{\text {mag }}=11.77 \pm 0.02$ on 23 January (MJD 57045), and then demonstrated rapid flux variations above $R_{\mathrm{mag}}=11.9$ (Spiridonova et al. 2015). In general, the optical light curves and the Swift-UVOT light curves show the same trend, a double peaked shape with the second peak coincident with the dashed 


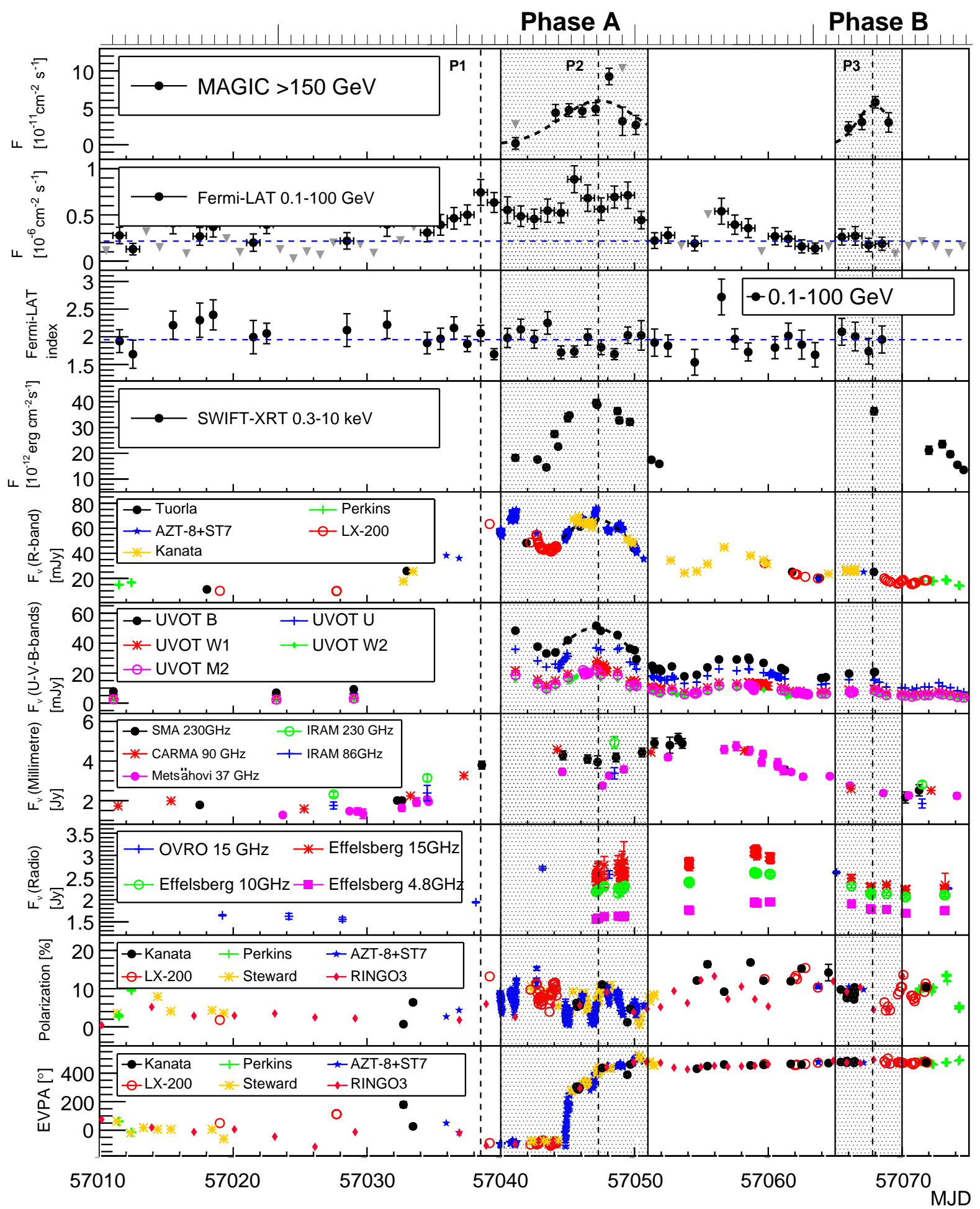

Fig. 1. Multi-wavelength flux and index curves of S5 0716+714 during the period from MJD 57010 to MJD 57080 (19 December 2014 to 27 February 2015). The shadowed areas indicate Phase A (from 18 to 27 January 2015 - MJD 57040 to MJD 57050) and Phase B (from 12 to 17 February 2015 - MJD 57065 to MJD 57070) high states in the VHE range and the corresponding activity in the other bands. P1, P2 and P3 (vertical dashed lines) indicate peaks in the HE and VHE emission.

vertical line $\mathrm{P} 2$ in Fig. 1. P2 is in fact identifying a peak not only in the VHE light curve, but also in the X-ray, optical, and $U V$ bands. The AZT- $8+$ ST7 light curve can be fit by a Gaussian peaking on MJD 57047 (25 January 2015) at a flux of $(67.49 \pm 0.01) \mathrm{mJy}$. The standard deviation of the fit is $\sigma=3.366 \pm 0.001$ days. The Swift-UVOT light curve can be fit by a Gaussian peaking on MJD 57047 (25 January 2015) at a flux of $(50.2 \pm 0.1) \mathrm{mJy}$. The standard deviation of the fit is $\sigma=3.36 \pm 0.02$ days. The activity in the radio band shown in Fig. 1 in Phase A and Phase B is moderate compared to 
Table 1. Summary of important dates.

\begin{tabular}{ccl}
\hline \hline MJD & Calendar date & Description \\
\hline 57038.5 & 16 January 2015 & P1: first peak of the HE emission $\rightarrow$ trigger VHE observations \\
57040 & 18 January 2015 & Start of Phase A \\
$57044 / 45$ & 22/23 January 2015 & 1 day EVPA rotation of 360 \\
$57047.3 \pm 0.53$ & 25 January 2015 & P2: Gaussian fit peak of the VHE emission in PHASE A \\
57050 & 28 January 2015 & End of Phase A \\
$57050 \pm 3$ & 28 January 2015 & K14b passage through A1 \\
57056 & 03 February 2015 & R4: Gaussian fit peak of radio emission in the intermediate phase \\
57065 & 12 February 2015 & Start of Phase B \\
$57067.8 \pm 0.23$ & 14 February 2015 & P3: Gaussian fit peak of the VHE emission in Phase B \\
57070 & 16 February 2015 & End of Phase B \\
57092 & 11 March 2015 & R5: Gaussian fit peak of radio emission \\
\hline
\end{tabular}

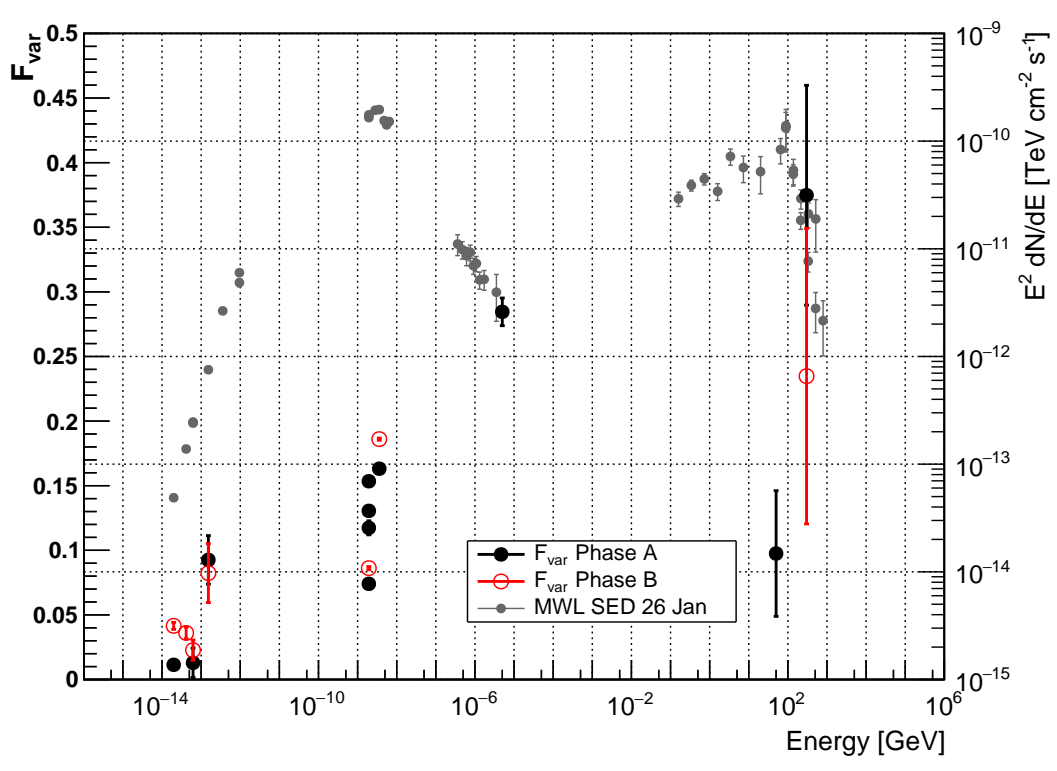

Fig. 2. Fractional variability $\left(F_{\mathrm{var}}\right)$ as a function of the energy for Phase A and Phase B. Vertical bars denote $1 \sigma$ uncertainties. In gray an SED of the source is overlayed (a snapshot of 26 January 2015) to facilitate association of the value of the fractional variability to the corresponding energy band. the other energy bands but does not describe a simply quiescent radio state. In the past the source has gone through many high states in the radio band, for instance the one described in Fig. 2 of Rani et al. (2013) named R6. During the R6 flare, the highest flux density reported was $\sim 10 \mathrm{Jy}$ while in the present one, the highest level of radio flux density at the same frequency $(230 \mathrm{GHz})$ is only $5 \mathrm{Jy}$. We study in more detail the possible delay between radio and optical $/ \gamma$-ray bands in Sect. 3.1.

As reported in Chandra et al. (2015a), the Phase A flare presents a double peaked shape in the HE $\gamma$-ray and optical bands. The feature is particularly evident in the $R$-band. The $\gamma$-ray light curve has the first sub-peak (in Fig. 1, corresponding to the P1 vertical dashed line) located immediately before Phase A at MJD 57038.5 (16 January 2015). That indicates the optical $/ \gamma$-ray emission as possible precursors of the VHE activity, whose peak starts to rise after MJD 57040 (18 January 2015) as indicated by the Gaussian fit of the VHE light curve in the top panel of Fig. 1. The Phase B flare is very different, being clearly visible in the VHE and X-ray band only. All the other bands are in a quiescent level, perhaps reproducing the conditions of the X-ray flare in December 2009 in Rani et al. (2013), where VHE data were not available. In that case, the $\mathrm{X}$-ray emission was described by both synchrotron and inverse Compton mechanisms in a single-zone, one-population leptonic model.
The fractional variability $F_{\text {var }}$ has been calculated using Eq. (10) in Vaughan et al. (2003):

$F_{\mathrm{var}}=\sqrt{\frac{S^{2}-\overline{\sigma_{\mathrm{err}}^{2}}}{\bar{x}^{2}}}$,

which represents the normalized excess variance. $S$ stands for the standard deviation and $\overline{\sigma_{\text {err }}^{2}}$ the mean square error of the flux measurements, while $\bar{x}$ indicates the average flux. The uncertainty of $F_{\text {var }}$ is given by Eq. (2) in Aleksić et al. (2015), after Poutanen et al. (2008). $F_{\text {var }}$ was calculated for all the light curves shown in Fig. 1 and the results are plotted in Fig. 2 for both Phase A (full black dots) and Phase B (red open circles). To make a direct comparison of the variability determined for the various energy bands, we computed $F_{\text {var }}$ using only the multi-instrument observations strictly simultaneous to those performed by MAGIC. The overall behavior of the fractional variability shows a rising tendency with increasing energy, at least up to the X-ray frequency. Since $F_{\text {var }}$ is highly sensitive to the sampling of the observed data, in the HE and VHE bands where the sampling is poorer, the results are affected by very large error bars, making it impossible to confirm a general trend of $F_{\text {var }}$ for the whole energy spectrum. The highest fractional variabilities in Phase A occur in the VHE $\gamma$-ray band and in the X-ray band, with MAGIC $\left(F_{\mathrm{var}}=0.37 \pm 0.08\right)$, 


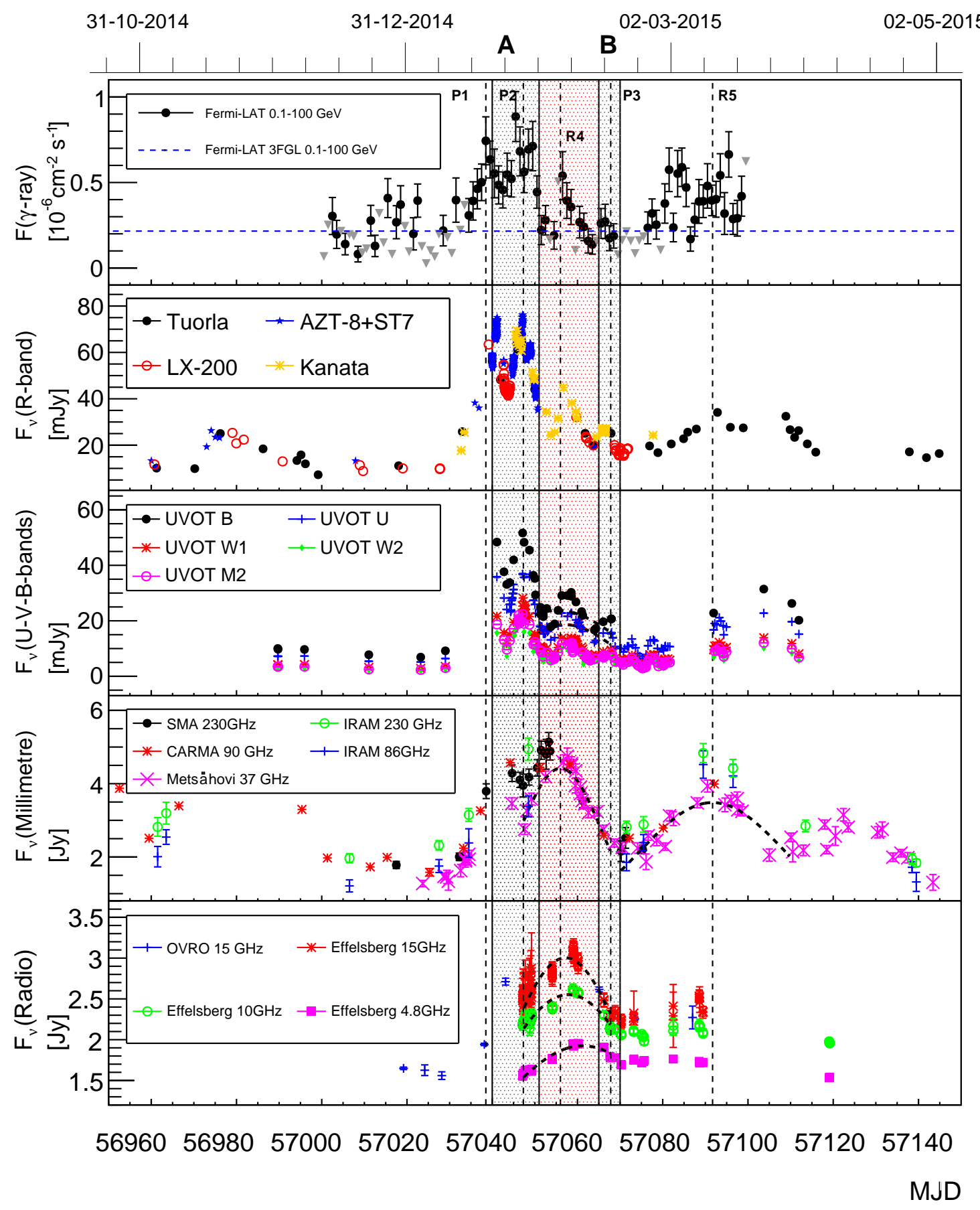

Fig. 3. HE $\gamma$-ray, optical, and radio activity of S5 0716+714 during the period from MJD 56950 to MJD 57150 (20 October 2014 to 08 May 2015). The three shadowed areas indicate (from the left side) Phase A (from 18 to 27 January 2015 - MJD 57040 to MJD 57050), an intermediate-phase (from 28 January to 11 February 2015 - MJD 57050 to MJD 57064), and Phase B (from 12 to 17 February 2015 - MJD 57065 to MJD 57070). Vertical dashed lines mark important dates, as shown in Table 1.

and Swift-XRT $\left(F_{\mathrm{var}}=0.28 \pm 0.010\right)$. In Phase B the highest fractional variability is again in the VHE range with MAGIC $\left(F_{\text {var }}=0.23 \pm 0.13\right)$, not far from the one obtained by UVOT telescope $\left(F_{\text {var }}=0.18 \pm 0.8 \times 10^{-3}\right)$.

\subsection{Analysis of radio activity in a larger time-frame}

When MAGIC detected S5 0716+714 for the first time in 2008 (Anderhub et al. 2009), the radio band was at a quiescent level. Here we see an increased activity in the low radio frequencies, especially in the intermediate period between Phases A and B. This activity could simply be an effect of a previous smaller flare in high energy delayed by months as seems typical for this source when considering longer periods of observation (Rani et al. 2013, 2014).

The present work includes one month of data, from the beginning of Phase A to the end of Phase B (MJD 57040 to MJD 57070). To gain a better understanding of the radio behavior of the source, we gathered $\gamma$-ray, optical, and radio data in Fig. 3, for a longer time-period of 8 months centered on 
28 January 2015 (MJD 57050). VHE emission was not detected outside of Phases A and B, but the data from the available instruments in the eight-month time window make it possible to better investigate the radio response and to compare the present dataset with the scenarios described in other previous multi-wavelength flares.

In Fig. 3, Phases A and B are still defined by gray shadowed areas, while the intermediate phase is filled in light red. From Fig. 3, second panel from the bottom, the radio activity in the intermediate zone between Phases A and B (from MJD 57051 to MJD 57065-29 January to 12 February 2015) could be fit by a Gaussian shape $\left(\chi^{2} /\right.$ n.d.f. $=34.9 / 14$ and standard deviation of $\sigma=9.7 \pm 0.3$ days), with a maximum flux density of $4.42 \pm 0.07 \mathrm{Jy}$, corresponding to MJD 57056 (03 February 2015).

In the bottom panel of Fig. 3, which shows the radio activity in lower frequencies from the Effelsberg telescope, a peak could be identified by a Gaussian fit of the data $\left(\chi^{2} /\right.$ n.d.f. $=28.7 / 47$ and standard deviation of $\sigma=14.5 \pm 0.5$ days), for a maximum flux density at $15 \mathrm{GHz}$ of $3.00 \pm 0.02 \mathrm{Jy}$, corresponding to MJD 57057 (04 February 2015). If we consider the dashed vertical line R4 as the position of the radio peak (using the value of MJD 57056 - 03 February 2015 retrieved from the Metsähovi data which has a smaller error), we can see a delay with respect to the $\gamma$-ray/optical peak P2 of $\sim 9$ days, which is considerably smaller than the delays of $\sim 65$ days or more found in Rani et al. (2014). Another hint of delayed activity, indicated by the dashed vertical line labeled as R5, can be seen in Metsähovi data, with a maximum flux density of $3.48 \pm 0.06$ Jy, corresponding to MJD 57092 (11 March 2015), fit by a Gaussian with $\chi^{2} /$ n.d.f. $=84.3 / 13$ and standard deviation of $\sigma=17.3 \pm 0.9$ days. In the latter case, the delay from the P 2 flare would be longer, $\sim 45$ days.

Based on data from April 2007 to January 2011, a considerable delay of the radio, $\sim 65$ days from the optical $/ \gamma$ flare, was found in Rani et al. (2013). In Rani et al. (2014), using a dataset from August 2008 to September 2013, the highest peak in radio flux occurred $\sim 82 \pm 32$ days after the $\gamma$-ray one. The delay in the radio emission from the optical $/ \gamma$ ones supports a scenario in which the $\gamma$-ray emission is produced upstream of the core while the radio emission has its origin in a shock in the jet, first appearing and evolving in the innermost, ultracompact VLBI core region, and subsequently moving downstream in the jet at parsec scales with apparent superluminal speeds. Similar results, on a larger sample of blazars, are presented in Fuhrmann et al. (2014). A longer-term study centered on the flaring activity reported here could be interesting for future investigations but is beyond the scope of the present work.

\subsection{Electric Vector Position Angle swing}

An important feature of Phase A is determined by the very fast change in the electric vector position angle happening over the night MJD 57044 (22 January 2015), 4 days after the first peak P1 in the optical band and 2 days before the MAGIC peak P2. This particular feature can be seen in the bottom panel of Fig. 1, as well as in Fig. 4, where the feature is zoomed in the time range MJD 57043-MJD 57048 (21-26 January 2015).

The dataset we present contains EVPA data coming from many different instruments, as shown in the bottom panel of Fig. 1. All the EVPA data collected are in agreement and were treated as in Larionov et al. (2013): in particular, to solve the $\pm 180^{\circ}$ ambiguity, we have added/subtracted $180^{\circ}$ each time that the subsequent value of EVPA was $>90^{\circ}$ less/more than the preceding one.

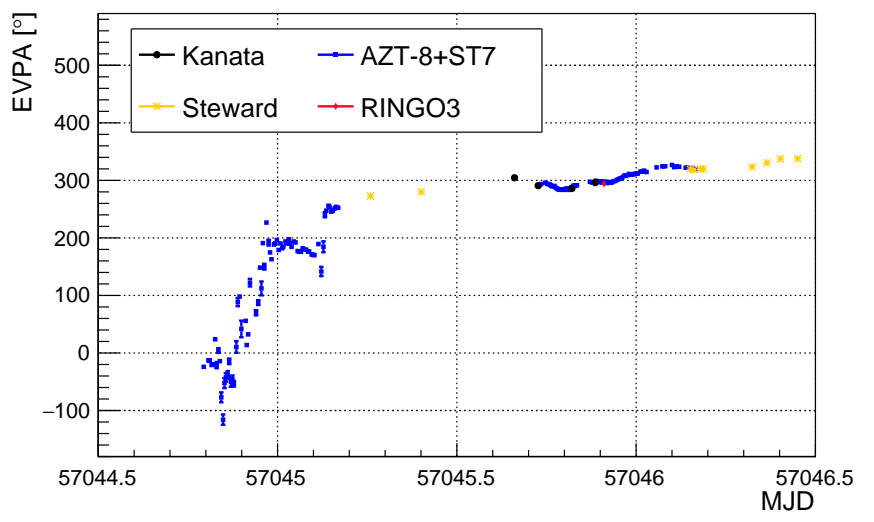

Fig. 4. Zoom of the EVPA rotation of S5 $0716+714$ during the period from MJD 57043 to MJD 57048 (21 to 26 January 2015) in Phase A.

In Marscher et al. (2008), a similar behavior of the EVPA was reported for BL Lacertae: a radio to $\gamma$-ray outburst, accompanied by a rotation of the EVPA, was observed as the consequence of a bright feature moving in the jet. In that case, the EVPA rotation was slower: it rotated steadily by about $240^{\circ}$ over a five-day interval before settling at a value of $195^{\circ}$.

In 2008, when the source was observed in the VHE range for the first time (Anderhub et al. 2009), the simultaneous optical outburst was accompanied by a $\sim 360^{\circ}$ PA rotation of the electric vector, as reported in Larionov et al. (2008a). The rotation happened with an approximate rate of $60^{\circ}$ per day, hence slower than in the present case. That rotation was interpreted as a consequence of the propagation of polarized emission from a knot spiraling down the jet. Chandra et al. (2015a) investigated the PA rotation swing from Phase A using the data from the Steward Observatory, in the frame of the HMFM (Zhang et al. 2014), suggesting that the fast rotation was due to reconnections in the emission region in the jet.

\section{Jet evolution study with very-long-baseline interferometry}

We analyzed the structure of the jet with very-long-baseline interferometry. Details of the analysis are presented in Sect. 2.5.

As shown in Fig. 5, the core of the jet is the brightest knot, designated $A 0$, which is the southern-most feature of the jet, and assumed to be stationary. The position of the core across epochs is shown by the black horizontal line. In addition to the core, we have identified a stationary feature, $A 1$, and two moving knots, K14a and K14b. Figure 6a shows the evolution of the positions of the knots. We note that a stationary feature at a position similar to that of $A 1$ was reported previously by Rani et al. (2015) and Jorstad et al. (2017) in data obtained from 2007 to 2013. The moving knots have apparent speeds of $(10.7 \pm 0.8) \mathrm{c}$ and $(9.7 \pm 1.8)$ c for K14a and K14b, respectively, although K14a decelerates at $\sim 0.5$ mas from the core. The direction of motion $\Phi$ is $\Phi_{\mathrm{K} 14 \mathrm{a}}=(25.4 \pm 2.2) \mathrm{deg}$ and $\Phi_{\mathrm{K} 14 \mathrm{~b}}=(43.3 \pm 5.6) \mathrm{deg}$, respectively. Extrapolation of the motion of K14a and K14b back to the VLBI core suggests that K14a and K14b passed through the core on MJD $56880 \pm 22$ and MJD $56971 \pm 30$, respectively.

Knot K14b is of special interest with respect to the highenergy event in January 2015 (MJD 57040-57050): according to its proper motion of $(0.51 \pm 0.09) \mathrm{mas} / \mathrm{yr}, \mathrm{K} 14 \mathrm{~b}$ passed through $A 1$ on MJD $57050 \pm 30$. This coincides with the high $\gamma$-ray state and $\mathrm{TeV}$ detection of S50716+714. Moreover, Fig. 6b shows that $A 1$ had an elevated flux density at the epoch closest to 


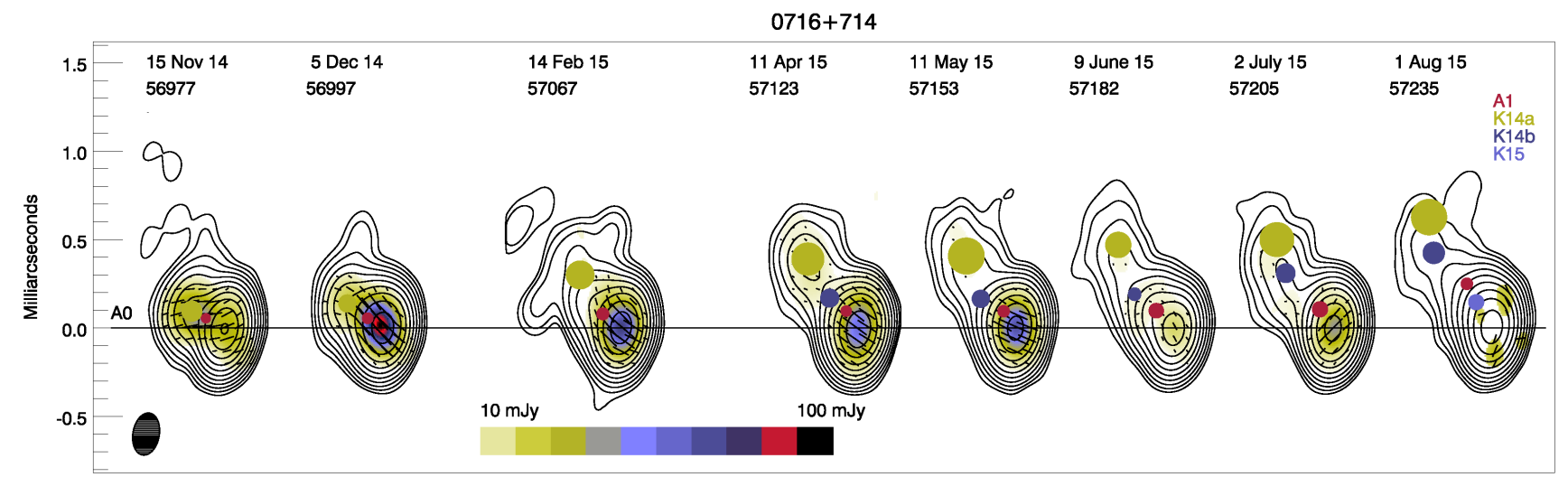

Fig. 5. A sequence of total (contours) and polarized (color scale) intensity images of S5 $0716+714$ at $43 \mathrm{GHz}$, convolved with a beam of $0.24 \times 0.15 \mathrm{mas}^{2}$ at $P A=-10^{\circ}$. The global total intensity peak is $2655 \mathrm{mJy} /$ beam and the global polarized intensity peak is $107 \mathrm{mJy} / \mathrm{beam}$; black line segments within each image show the direction of polarization; the black horizontal line indicates the position of the core, A0.
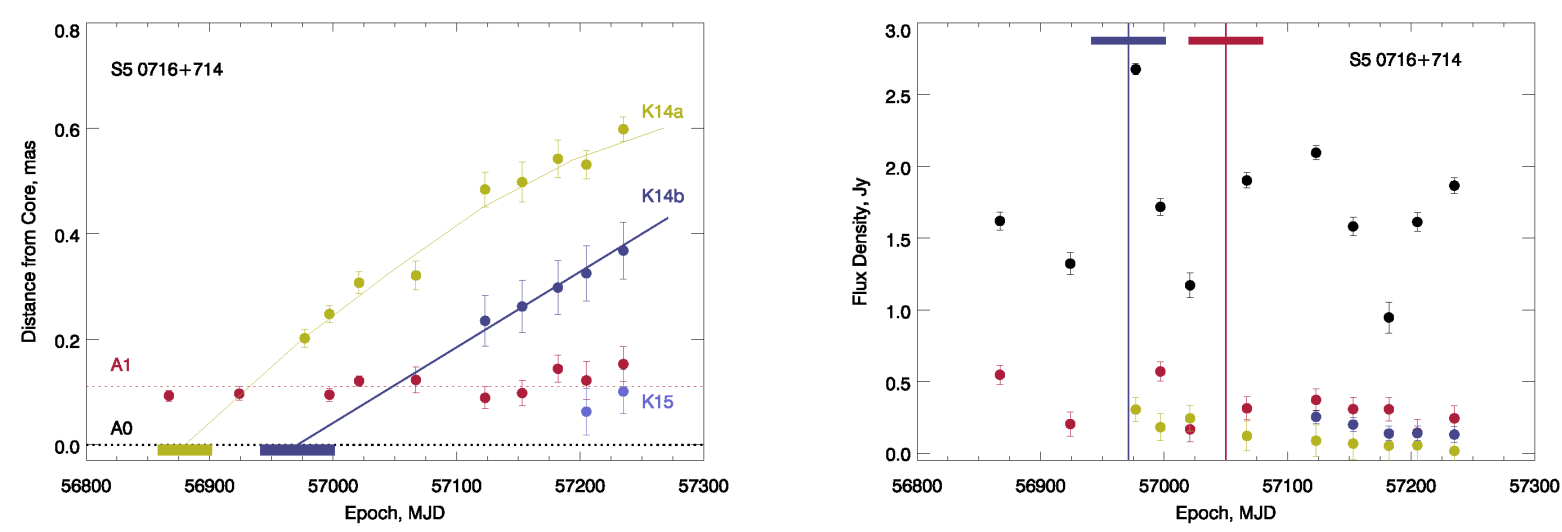

Fig. 6. VLBA-BU-BLAZAR analysis of S5 0716+714. Left panel: separation of knots A1 (red), K14a (yellow), K14b (blue), and K15 (light blue) from the core $A 0$ (black dotted line); the yellow and blue line segments on the position of $A 0$ indicate the $1 \sigma$ uncertainty of the ejection times of K14a and K14b, respectively. Right panel: light curves of the core $A 0$ (black), stationary feature $A 1$ (red), and moving knots K14a (yellow) and $\mathrm{K} 14 \mathrm{~b}$ (blue); flux densities of K14b are shifted by $\sim 0.1 \mathrm{Jy}$ for clarity; vertical blue and red lines indicate time of passage of K14b through $A 0$ and $A 1$, respectively.

MJD 57050 when K14b should have been passing through $A 1$ according to its proper motion. In addition, a change of the position angle of $A 1$ from $\sim 80^{\circ}$ to $\sim 50^{\circ}$ is detected around the time of the expected passage (see Table A.1). The latter angle is close to the PA of $\mathrm{K} 14 \mathrm{~b}, \Theta=(47.5 \pm 4.6) \mathrm{deg}$. This implies an interaction between superluminal knot K14b and stationary feature $A 1$, and supports the hypothesis that $A 1$ is a recollimation shock similar to that observed in BL Lacertae, for example (Marscher et al. 2008; Cohen et al. 2014).

In addition, the average size of $A 1$ is $(0.049 \pm 0.020)$ mas, which implies that $\mathrm{K} 14 \mathrm{~b}$ needs $(35 \pm 13)$ days to pass through the stationary feature. The latter agrees very well with the duration of 34 days of the elevated $\gamma$-ray flux in the Fermi light curve of S5 0716+714, from MJD 57032 to 57066 (10 January 2015 to 13 February 2015). In this scenario, the $\mathrm{TeV}$ detections can be associated with the entrance and exit of the superluminal knot in and out of the recollimation shock.

\section{Spectral fitting of NuSTAR and Swift-XRT data}

For NUSTAR data, we performed the spectral fitting with XSPEC v12.8.2, with the standard instrumental response matrices and effective area files derived using the ftool nuproducts. We fit the data for both NUSTAR detectors simultaneously, allowing an offset of the normalization factor for module FPMB with respect to module FPMA. Regardless of the adopted models, the normalization offset was less than 5\%. First, we adopted a simple power-law model modified by the effects of the Galactic absorption, corresponding to a column of $3.11 \times 10^{20} \mathrm{~cm}^{-2}$ (Kalberla et al. 2005). The fit returns the power-law index of $1.93 \pm 0.04$, but the residuals show that the NUSTAR spectrum is more concave (i.e., the spectrum becomes flatter towards higher energies) than a simple powerlaw model would imply. In addition, this index is significantly harder than that inferred from the Swift-XRT data alone (which shows the index of $\sim 2.75$ ), which also suggests a more complex spectral model.

Since the NUSTAR and Swift data were nearly strictly contemporaneous (with a significant overlap) we fit the NuSTAR and Swift-XRT data simultaneously, but allowing for the normalizations of Swift and NUSTAR to fit independently. We attempted two more complex models (both with absorption fixed at the Galactic value as above). First, we considered a broken power law, with steeper low-energy and harder high-energy indices. This is similar to the model considered by Wierzcholska \& Siejkowski (2016). The low- and highenergy indices are, respectively, $2.52 \pm 0.07$ and $1.81 \pm 0.08$, the break energy is at $5.2_{-0.5}^{+0.7} \mathrm{keV}$, and $\chi^{2}$ is 351 for $328 \mathrm{PHA}$ 
channels. We note that the break energy in our fit is somewhat lower than that determined by Wierzcholska \& Siejkowski (2016), but this is likely due to a different choice of bandpass, size of the source extraction region, and precise location of the region of the detector from which the background was subtracted.

We also attempted a double power-law representation of the data, also modified by Galactic absorption as above: here, the resulting spectrum is a sum of two power-law models, and is probably more physically motivated than a broken power law. The fit returns $\chi^{2}=352$ for 328 PHA bins with a low-energy index of $2.62 \pm 0.16$ and a high-energy index of $1.41 \pm 0.22$. Since this model can represent a physically sensible superposition of two separate components, we express a preference for the twopower-law spectral form. With this model, the $2-10 \mathrm{keV}$ flux is $(9.7 \pm 0.7) \times 10^{-12} \mathrm{erg} \mathrm{cm}^{-2} \mathrm{~s}^{-1}$. We note here that the most reasonable interpretation of such a two-component spectral shape is that we are witnessing a contribution of two separate components, namely the "tail" of the low-energy component (presumably produced by the synchrotron process) and the onset of the high-energy component (presumably due to the IC process). We plot the unfolded spectrum of the Swift-XRT and NuSTAR data observed on 24 January 2015 (MJD 57046) and fit to the twopower-law model in Fig. 7.

\section{VHE differential energy spectrum and EBL deabsorbtion}

The VHE $\gamma$-rays from distant blazars can interact with the optical-UV photons from the extragalactic background light (EBL; Gould \& Schreder 1967; Stecker et al. 1992) via pair production, resulting in an attenuation of the intrinsic VHE spectrum. Finite resolution of the instrument will also modify the intrinsic spectrum. Unfolding techniques are adopted in the MARS code to unfold the observed spectrum from the instrument response. The differential spectrum of S50716+714 is shown in Fig. 8 for a simple unfolding considering instrumental response only (hereafter, "observed spectrum"). An unfolding, including de-absorption from EBL with the Domínguez et al. (2011) model (hereafter the intrinsic spectrum), was also performed, and parameters of the observed and intrinsic spectra are reported in Table 2. The EBL imprint on the $\gamma$-ray spectra from distant blazars could be used to constrain the EBL density, under some assumptions on the intrinsic spectrum of the source (see e.g., Ackermann et al. 2012; Abramowski et al. 2013).

The differential VHE spectra, observed as well as EBLcorrected using the model of Domínguez et al. (2011), can be described by a power-law:

$\frac{\mathrm{d} F}{\mathrm{~d} E}=f_{0}\left(\frac{E}{150 \mathrm{GeV}}\right)^{-\Gamma}$,

where the normalization constant $f_{0}$, the spectral index $\Gamma$, the goodness of the fit $\left(\chi^{2} /\right.$ n.d.f. and probability $P$ ), and the energy range of the fit $E$ are indicated in Table 2 for data from Phases $A$ and $\mathrm{B}$.

\section{Redshift estimation}

The simultaneous spectra from MAGIC and Fermi-LAT were used to estimate the redshift of the source. We apply the method presented in Prandini et al. (2010, 2011) based on the assumption that the slope of the VHE spectrum corrected for EBL absorption should not be harder than the one measured by FermiLAT at lower energies. The redshift at which the two slopes

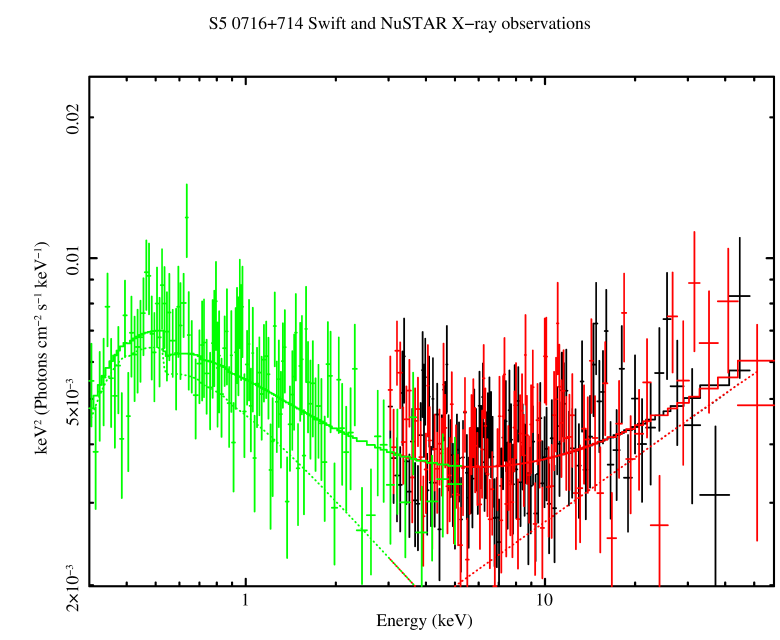

Fig. 7. Unfolded X-ray spectrum of S50716+714 derived from simultaneous fitting of the contemporaneous Swift-XRT and NuSTAR data obtained on 24 January 2015 (MJD 57046). The adopted model is a sum of two power laws. Swift-XRT data are plotted in green, while the two NUSTAR modules are plotted in red and black, respectively. The "valley" between the two main broad-band spectral peaks is in the X-ray band.

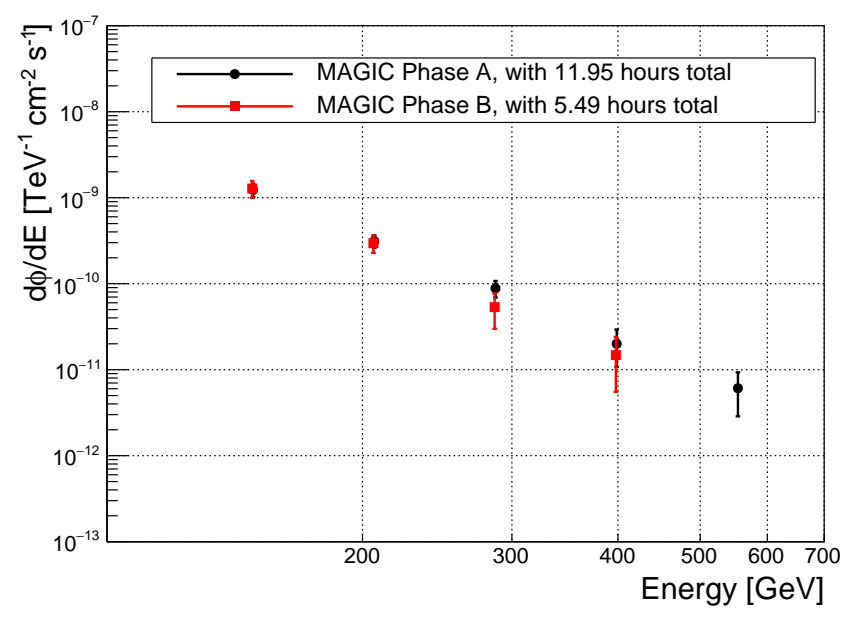

Fig. 8. Unfolded observed differential energetic spectra by MAGIC for Phase A (black full dots) and Phase B (red full squares). Parameters for the spectra (including the ones regarding the intrinsic EBL deabsorbed spectra with Domínguez et al. 2011 model) are reported in Table 2.

match, $z^{\star}$, is then used as an upper limit estimate of the source distance if there is no spectroscopic redshift available. If we apply this method to the data presented here, assuming the Franceschini et al. (2008) EBL model, a $2 \sigma$ upper limit on the redshift of 0.598 is found.

The empirical formula proposed in Prandini et al. (2011) applied to this data gives $z_{\text {rec }}=0.31 \pm 0.02_{\text {stat }} \pm 0.05_{\text {sys }}$ as the most probable value for the redshift, where the first error is related to the statistical errors of Fermi-LAT and MAGIC slopes, while the second error is the error of the method itself, as estimated in Prandini et al. (2011). This value is in agreement with those given by Nilsson et al. (2008) and Danforth et al. (2013). The value of $z=0.31 \pm 0.08$ found in Nilsson et al. (2008) was based on the photometric detection of the host galaxy, while the $z<0.322$ (95\% confidence) result reported by Danforth et al. (2013) was obtained by detection of Ly- $\alpha$ systems in the ultraviolet spectrum of the source. For the SED modelling (see 
Table 2. VHE spectrum parameters for a PWL fit.

\begin{tabular}{lccccc}
\hline \hline \multicolumn{1}{c}{$f_{0}\left(\mathrm{~cm}^{-2} \mathrm{~s}^{-1} \mathrm{TeV}^{-1}\right)$} & $\Gamma$ & $\chi^{2} /$ n.d.f. & $P$ & $E(\mathrm{GeV})$ \\
\hline \multicolumn{7}{c}{-Phase A- } \\
\hline Observed & $\left(2.75 \pm 0.25_{\text {stat }} \pm 0.30_{\text {sys }}\right) \times 10^{-10}$ & $4.08 \pm 0.22_{\text {stat }} \pm 0.15_{\text {sys }}$ & $0.11 / 3$ & 0.99 & $127.2-659.1$ \\
Intrinsic & $\left(4.85 \pm 0.45_{\text {stat }} \pm 0.53_{\text {sys }}\right) \times 10^{-10}$ & $2.73 \pm 0.28_{\text {stat }} \pm 0.15_{\text {sys }}$ & $1.68 / 3$ & 0.64 & $127.2-659.1$ \\
\hline \multicolumn{7}{c}{ - Phase B - } \\
\hline Observed & $\left(7.94 \pm 1.27_{\text {stat }} \pm 0.87_{\text {sys }}\right) \times 10^{-10}$ & $4.64 \pm 0.49_{\text {stat }} \pm 0.15_{\text {sys }}$ & $0.17 / 2$ & 0.92 & $127.2-474.3$ \\
Intrinsic & $\left(1.06 \pm 0.17_{\text {stat }} \pm 0.11_{\text {sys }}\right) \times 10^{-10}$ & $3.65 \pm 0.53_{\text {stat }} \pm 0.15_{\text {sys }}$ & $0.23 / 2$ & 0.89 & $127.2-474.3$ \\
\hline
\end{tabular}

following section), we used the redshift value of 0.26 as in Anderhub et al. (2009). This value is within the errorbars of the redshift determined here as well as within other observations (see the introduction).

\section{Broadband SED}

The multi-wavelength SEDs for Phases A and B are presented in Fig. 9. Archival data from ASDC (ASI Science Data Center) ${ }^{13}$ are shown in gray. When the source was detected for the first time in the VHE range (Anderhub et al. 2009) by MAGIC, the only available simultaneous multi-wavelength data were coming from KVA (optical) and Swift (X-rays) and there were no constraints on the second bump beyond the MAGIC data. Nevertheless, the very soft X-ray spectra belonging to the synchrotron component, combined with the high VHE $\gamma$-ray flux challenged the simple one-zone SSC model as it would require a very high flux of $\gamma$-rays around $10 \mathrm{GeV}$, higher than has been observed from the source with Fermi-LAT or its precursor EGRET. This condition for onezone persists also for the new data, but now we actually have simultaneous data in this energy range from Fermi-LAT and we find that we cannot describe the observed broadband SED with the one-zone SSC model during this flaring period.

While a one-zone SSC model can match the observed Swift$\mathrm{XRT}+\mathrm{N} U \mathrm{STAR}$ spectrum as a transition between synchrotron and IC components, and simultaneously the $\gamma$-ray data from Fermi-LAT and MAGIC, it tends to under-reproduce the observed optical flux. This has been independently verified using the BLAZAR code (Moderski et al. 2003).

Based on the multiwavelength data in Sect. 3 and VLBA data in Sect. 4, we use a two-zone model to describe the SED in Phases A and B. We use two blobs close to each other to represent a situation where a superluminal knot (blob 1) is interacting with a recollimation shock region (blob 2). The Phase A SED represents a snapshot of a time when the knot enters the recollimation shock region and the Phase B SED represents a time when the knot has exited the recollimation region. We model the two blobs with a framework similar to one presented for flat-spectrum radio quasar PKS $1222+216$ in Tavecchio et al. (2011), modified for the case of no external seed photons as in Aleksić et al. (2014) for PKS 1424+240. Unlike the case of PKS $1424+240$, in our case the two emission regions are very close to each other and they provide seed photons for inverse Compton scattering for each other. The model blobs have broken power-law electron spectra $\left(\gamma_{\min }, \gamma_{\mathrm{b}}\right.$ and $\gamma_{\max }$ are the minimum, break, and maximum Lorentz factors respectively; $n_{1}$ and $n_{2}$ are the low- and high-energy slope of the smoothed

13 http://www .asdc.asi.it/ power law electron energy distribution), magnetic field $B$, normalization of the electron distribution $K$, radius of the emission region $R$ and Doppler factor $\delta$.

We also use the observed variability behavior as a guide on how the parameters change between Phases A and B. As discussed in Sect. 3, Phases A and B have different variability behaviors; while Phase A consists of a flare in all bands, in Phase B the activity is constrained to the X-ray and VHE $\gamma$-ray bands. To limit the number of free parameters, we fix the larger component (which is representing the recollimation shock) to have mostly the same parameters in Phases A and B. We only change $K$ to a lower value to represent the general lower state in optical and $\mathrm{GeV} \gamma$-rays of Phase B. We then find parameters for the smaller emission region to describe the observed SEDs in Phases A and B separately. In both panels of Fig. 9, the "blob1" component is represented by the red dashed line. The "blob2" component of the model is represented by the blue dash-dotted line in both panels, and the emission resulting from the interaction of the two components is reported with a green line.

We report a set of parameters we found to give a reasonable description (but see below) of the observed SED in Table 3 for both phases. The set of parameters we present is not unique, but the parameters used are within the range typically found for $\mathrm{TeV}$ blazars and also for the ones found for PKS 1424+240 using the similar modeling setup.

Even if this simple two-zone model provides a better representation of the observed data from radio to VHE $\gamma$-rays with respect to a one-zone model, the model line is slightly lower than the $\gamma$-ray fluxes in the range of $10-100 \mathrm{GeV}$, albeit within the systematic uncertainties of the data. The data in this energy range suggests a rather sharp feature, which is impossible to reproduce with the simplistic model that we used. In general, sharp features require the presence of external seed photons such as those used by Böttcher et al. (2013), for example, to model the SED of the source in a lower state. However, there is no observational evidence for such an external seed photon field from optical spectroscopy nor from the scenario we presented for the flaring behavior within this epoch; therefore no such component was added to the modeling.

There are also other possible two-zone model setups, such as a spine-sheath model (Ghisellini et al. 2005), where a slower sheath of the jet surrounds a faster spine. For the previous VHE $\gamma$-ray flaring epoch, a spine-sheath model (Ghisellini et al. 2005) was shown to provide a reasonable fit to the SED data (Anderhub et al. 2009; Tavecchio \& Ghisellini 2009). We tested the spine-sheath model for the SEDs shown here and found acceptable agreement with the SED data. This emphasizes that SED data alone are not enough to separate different two-zone models, but must be combined with constraints from VLBA and light curve variability. 

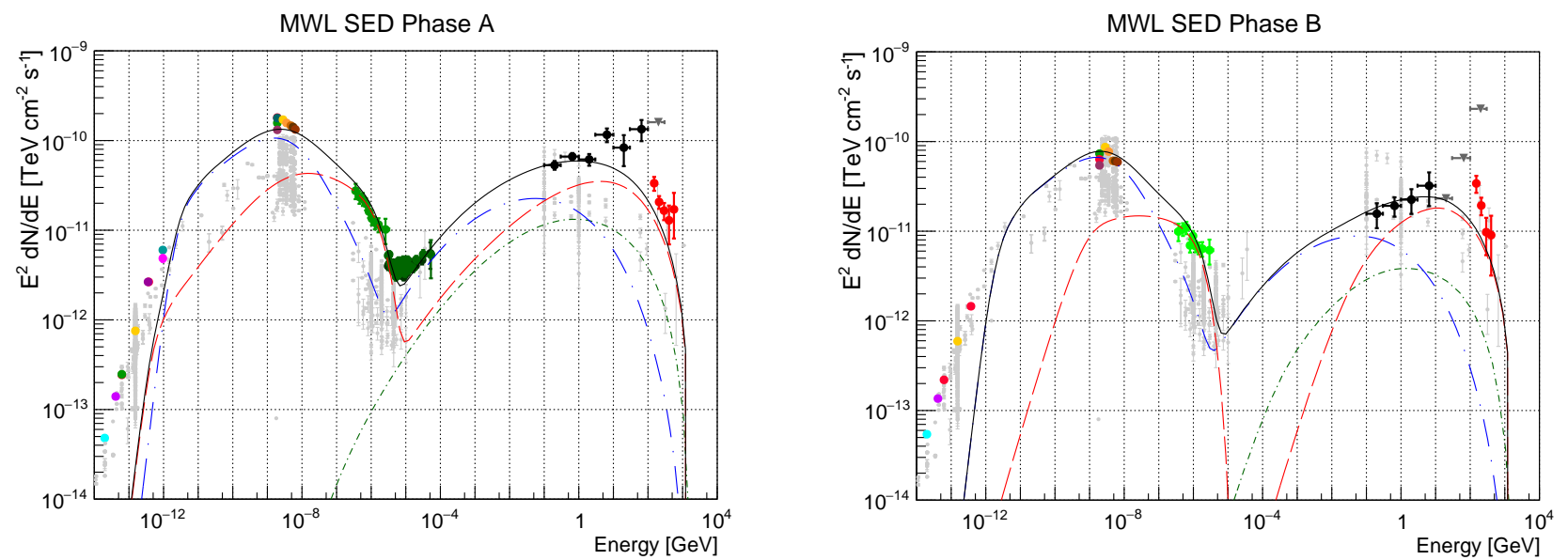

Fig. 9. MWL SEDs for Phases A and B. Archival data from ASDC are shown in gray. The two components (blobs representing a moving emission feature and a recollimation shock, see text) are shown with blue and red dashed lines. The green line is the emission that is a result of the interaction between these two blobs and the black solid line is the sum of these three components. The red full circles represent the intrinsic (EBL deabsorbed according to Domínguez et al. 2011) MAGIC SED used in the model. For data taken in the radio and optical bands, the error bars are smaller than the size of the marker.

Table 3. Input parameters for the emission models of S5 0716+714.

\begin{tabular}{lcccccccccc}
\hline \hline & $\gamma_{\min }$ & $\gamma_{\mathrm{b}}$ & $\gamma_{\max }$ & $n_{1}$ & $n_{2}$ & $B(\mathrm{G})$ & $K$ & $R(\mathrm{~cm})$ & $\delta$ & $z$ \\
\hline \multicolumn{8}{c}{-Phase A- } \\
\hline "blob1" & 100 & $1.3 \times 10^{4}$ & $2 \times 10^{5}$ & 1.95 & 3.5 & 0.1 & $3.5 \times 10^{3}$ & $2.6 \times 10^{16}$ & 25 & 0.26 \\
"blob2" & 300 & $1 \times 10^{4}$ & $1.5 \times 10^{5}$ & 2.32 & 4.6 & 0.12 & $1.6 \times 10^{4}$ & $6.5 \times 10^{16}$ & 25 & 0.26 \\
\hline \multicolumn{8}{c}{ - Phase B - } \\
\hline "blob1" & $4 \times 10^{3}$ & $8 \times 10^{3}$ & $2 \times 10^{5}$ & 1.9 & 3.2 & 0.09 & $9 \times 10^{3}$ & $1.35 \times 10^{16}$ & 25 & 0.26 \\
"blob2" & 300 & $1 \times 10^{4}$ & $1.5 \times 10^{5}$ & 2.32 & 4.6 & 0.12 & $1 \times 10^{4}$ & $6.5 \times 10^{16}$ & 25 & 0.26 \\
\hline
\end{tabular}

According to the scenario described above, which is supported by the dedicated VLBI study we performed in Sect. 4, we explain the extremely fast rotation of $\sim 360^{\circ}$ as produced by turbulence in the interaction between a superluminal knot and a stationary feature near the core. Being dependent on the orientation of the shock and the magnetic field threading it, EVPA provides a unique tool to understand the acceleration mechanisms and behavior of the shocked plasma. Recent studies on EVPA swings larger than $180^{\circ}$ simultaneous with HE $\gamma$-ray emission (Marscher et al. 2008, 2010; Abdo et al. 2010b) have been interpreted as additional evidence for a helical magnetic field structure. The existing models focusing on the description of the synchrotron polarization features (e.g., Lyutikov et al. 2005) apply a simple and time-independent power-law electron spectrum not taking into account possible predictions for the resulting HE emission. Our model, on the other hand, does not include a detailed geometry of the magnetic field or the angledependent synchrotron emissivity and polarization. Currently, only two models are able to represent the SED of blazars together with their synchrotron polarization features, including rotations of the EVPA: the HMFM (Zhang et al. 2014) and the turbulent, extreme multi-zone (TEMZ; Marscher 2014). In the HMFM, large polarization angle rotations by $\gtrsim 180^{\circ}$ are explained with the passage of a moving shock through a region with a highly disordered field; the compression of the shock orders the field partially, but this ordering is seen at different depths as time advances owing to light-travel delays. This leads to an apparent rotation of the polarization of $180^{\circ}$ per shock. In the
TEMZ model, randomness in the magnetic field direction in different turbulent cells can cause the observed rotations in the linear polarization vector, even as fast as the one observed in our case. Turbulence in general gives, at different times, "clusters" with small EVPA variation, relatively smooth EVPA rotations, step-wise EVPA changes, and random fluctuations. The behavior of S5 0716+714 in Phase A is consistent with this scenario.

\section{Summary and conclusions}

The BL Lac object S5 0716+714 has been studied in a multiwavelength frame from radio to the VHE $\gamma$-ray band. In January 2015, an unprecedented outburst of S5 0716+714 was registered in all energy bands, from low-frequency radio to VHE, and after almost a month, another high state was detected by the MAGIC and Swift-XRT instruments only. We divide the data into two phases (Phase A from 18 to 27 January 2015 - MJD 57040 to MJD 57050, and Phase B from 12 to 17 February 2015 MJD 57065 to MJD 57070) that represent very different characteristics, allowing a deep study of the broadband SEDs.

The broadband flaring activity period of Phase A coincides with the passage of a moving feature through a stationary feature (at $\sim 0.1 \mathrm{mas}$ ). We have found a very fast change in the EVPA during Phase A. The $>400$ deg swing in the optical EVPA is explained here as the passage of a superluminal knot through a stationary feature near the radio core. The VHE emission is then found originating in the entrance and exit of a superluminal knot in and out of a recollimation shock in the inner jet. This suggests 
that shock-shock interaction in the jet seems to be responsible for the observed flares and EVPA swing.

The jet behavior, studied with VLBA-BU-BLAZAR data, is in agreement with the scenario described in Rani et al. (2015), suggesting a connection between jet kinematics and the observed broadband flaring activity. More precisely, the $\gamma$-ray emission in the HE and VHE bands is attributed to a shock in the helical jet downstream of the core, closely followed by an optical and X-ray outburst in the core. The presence of low radio activity, observed during Phase A, was not reported in April 2008 when MAGIC observed the source, for the first time in the VHE range (Anderhub et al. 2009). In this case it could be a delayed response of a previous, less intense flare, resembling the behavior of the same source in the radio band reported in Rani et al. $(2013,2014)$, when simultaneous optical and/or $\gamma$ flares lagged behind the radio counterparts by almost two months.

The first peak in the VHE $\gamma$-ray emission takes place $\sim 2$ days after the very fast EVPA rotation and the second $\sim 18$ days after the new knot has emerged from the VLBA core. This is a strong indication that the VHE $\gamma$-ray emission is associated to a component entering and exiting the core region.

The broadband SEDs, including MAGIC and Fermi-LAT simultaneous data and the quasi-simultaneous NuStar data for the first time, could not be described by a simple one-zone model. Instead we used a two-zone model, where two spherical blobs are co-spatial and provide seed photons to each other. This modeling setup provides an acceptable description of the SEDs in Phases A and B, even if it is certainly an over-simplified representation of the true physical processes taking place when superluminal knots enter and exit the recollimation shock region.

Finally, we also investigated the redshift of S5 0716+714 Using simultaneous data from MAGIC and Fermi-LAT, the redshift was calculated to be $z=0.31 \pm 0.02_{\text {stat }} \pm 0.05_{\text {sys }}$, confirming the value present in the literature based on the photometric detection of the host galaxy (Nilsson et al. 2008) and the more recent upper limit from a direct detection (Danforth et al. 2013).

$\mathrm{S} 50716+714$ is an intermediate BL Lac object, and only a handful of these sources have been detected in VHE $\gamma$-rays. In almost all detections of VHE $\gamma$-rays, activity in other bands (optical and/or HE $\gamma$-rays) has been seen, but our very comprehensive dataset provided a unique insight into how these VHE $\gamma$-ray flares are connected to the activity in the jet.

At the end of December 2017, S50716+714 was flaring again in VHE $\gamma$-rays (Mirzoyan 2017). It will be interesting to see if the recognized patterns repeat also during this ongoing flaring period. This will be studied in a future paper.

Acknowledgements. We would like to thank the Instituto de Astrofísica de Canarias for the excellent working conditions at the Observatorio del Roque de los Muchachos in La Palma. The financial support of the German BMBF and MPG, the Italian INFN and INAF, the Swiss National Fund SNF, the ERDF under the Spanish MINECO (FPA2015-69818-P, FPA2012-36668, FPA2015 68378-P, FPA2015-69210-C6-2-R, FPA2015-69210-C6-4-R, FPA2015-69210 C6-6-R, AYA2015-71042-P, AYA2016-76012-C3-1-P, ESP2015-71662-C2-2-P, CSD2009-00064), and the Japanese JSPS and MEXT is gratefully acknowledged. This work was also supported by the Spanish Centro de Excelencia "Severo Ochoa" SEV-2012-0234 and SEV-2015-0548, and Unidad de Excelencia "María de Maeztu" MDM-2014-0369, by the Croatian Science Foundation (HrZZ) Project IP-2016-06-9782 and the University of Rijeka Project 13.12.1.3.02, by the DFG Collaborative Research Centers SFB823/C4 and SFB876/C3, the Polish National Research Centre grant UMO2016/22/M/ST9/00382 and by the Brazilian MCTIC, CNPq and FAPERJ. The Fermi-LAT Collaboration acknowledges generous ongoing support from a number of agencies and institutes that have supported both the development and the operation of the LAT as well as scientific data analysis. These include the National Aeronautics and Space Administration and the Department of Energy in the United States, the Commissariat à l'Énergie Atomique and the Centre
National de la Recherche Scientifique/Institut National de Physique Nucléaire et de Physique des Particules in France, the Agenzia Spaziale Italiana and the Istituto Nazionale di Fisica Nucleare in Italy, the Ministry of Education, Culture, Sports, Science and Technology (MEXT), High Energy Accelerator Research Organization (KEK) and Japan Aerospace Exploration Agency (JAXA) in Japan, and the K. A. Wallenberg Foundation, the Swedish Research Council and the Swedish National Space Board in Sweden. Additional support for science analysis during the operations phase is gratefully acknowledged from the Istituto Nazionale di Astrofisica in Italy and the Centre National d'Études Spatiales in France. This research was supported by an appointment to the NASA Postdoctoral Program at the Goddard Space Flight Center, administered by Universities Space Research Association through a contract with NASA. We thank the Swift team duty scientists and science planners. The Metsähovi team acknowledges the support from the Academy of Finland to our observing projects (numbers 212656, 210338, 121148, and others). The VLBA is an instrument of the Long Baseline Observatory. The Long Baseline Observatory is a facility of the National Science Foundation operated by Associated Universities, Inc. The Submillimeter Array is a joint project between the Smithsonian Astrophysical Observatory and the Academia Sinica Institute of Astronomy and Astrophysics and is funded by the Smithsonian Institution and the Academia Sinica. The OVRO 40$\mathrm{m}$ monitoring program is supported in part by NASA grants NNX08AW31G, NNX11A043G and NNX14AQ89G, and NSF grants AST-0808050 and AST-1109911. The St. Petersburg University team acknowledges support from Russian Science Foundation grant 17-12-01029. The BU group acknowledges support by NASA under Fermi Guest Investigator grant NNX14AQ58G and by NSF under grant AST-1615796. Part of this work was done with funding by the UK Space Agency. The VLBA is an instrument of the National Radio Astronomy Observatory. The National Radio Astronomy Observatory is a facility of the National Science Foundation operated under cooperative agreement by Associated Universities, Inc. The PRISM (Perkins Re-Imaging SysteM) camera at Lowell Observatory was developed by K. Janes et al. at BU and Lowell Observatory, with funding from the NSF, BU, and Lowell Observatory. This paper makes use of data obtained with the $100 \mathrm{~m}$ Effelsberg radio-telescope, which is operated by the Max-Planck-Institut für Radioastronomy (MPIfR) in Bonn (Germany). Part of this work is based on archival data, software or online services provided by the ASI Science Data Center (ASDC). PYRAF is a product of the Space Telescope Science Institute, which is operated by AURA for NASA. This paper is partly based on observations carried out with the IRAM $30 \mathrm{~m}$. IRAM is supported by INSU/CNRS (France), MPG (Germany) and IGN (Spain). IA acknowledges support by a Ramon y Cajal grant of the Ministerio de Economía, Industría y Competitividad (MINECO) of Spain. The research at the IAA-CSIC was supported in part by the MINECO through grants AYA2016-80889-P, AYA2013-40825$\mathrm{P}$, and AYA2010-14844, and by the regional government of Andalucia through grant P09-FQM-4784. The Liverpool Telescope is operated by JMU with financial support from the UK-STFC.

\section{References}

Abdo, A. A., Ackermann, M., Ajello, M., et al. 2010a, ApJ, 710, 1271 Abdo, A. A., Ackermann, M., Ajello, M., et al. 2010b, Nature, 463, 919 Abramowski, A., Acero, F., Aharonian, F., et al. 2013, A\&A, 550, A4 Acciari, V. A., Aliu, E., Aune, T., et al. 2009, ApJ, 707, 612

Acero, F., Ackermann, M., Ajello, M., et al. 2015, ApJS, 218, 23 Ackermann, M., Ajello, M., Allafort, A., et al. 2011, ApJ, 743, 171 Ackermann, M., Ajello, M., Albert, A., et al. 2012, Science, 338, 1190 Agudo, I., Thum, C., Molina, S. N., et al. 2018a, MNRAS, 474, 1427 Agudo, I., Thum, C., Ramakrishnan, V., et al. 2018b, MNRAS, 473, 1850 Akitaya, H., Moritani, Y., Ui, T., et al. 2014, Proc. SPIE, 9147, 914740 Aleksić, J., Ansoldi, S., Antonelli, L. A., et al. 2014, A\&A, 567, A135 Aleksić, J., Ansoldi, S., Antonelli, L. A., et al. 2015, A\&A, 573, A50 Aleksić, J., Ansoldi, S., Antonelli, L. A., et al. 2016, Astropart. Phys., 72, 76 Anderhub, H., Antonelli, L. A., Antoranz, P., et al. 2009, ApJ, 704, L129 Angelakis, E., Fuhrmann, L., Marchili, N., et al. 2015, A\&A, 575, A55 Antonucci, R. R. J., Hickson, P., Olszewski, E. W., \& Miller, J. S. 1986, AJ, 92, 1 Atwood, W. B., Abdo, A. A., Ackermann, M., et al. 2009, ApJ, 697, 1071 Baars, J. W. M., Genzel, R., Paulini-Toth, I. I. K., \& Witzel, A. 1977, A\&A, 61, 99 Bach, U., Krichbaum, T. P., Ros, E., et al. 2005, A\&A, 433, 815

Bachev, R., \& Strigachev, A. 2015, ATel, 6957

Böttcher, M., Reimer, A., Sweeney, K., \& Prakash, A. 2013, ApJ, 768, 54 Britzen, S., Kam, V. A., Witzel, A., et al. 2009, A\&A, 508, 1205 Breeveld, A. A., Landsman, W., Holland S. T., et al. 2011, AIP Conf. Proc., 1358, 373

Burrows, D. N., Hill, J. E., Nousek, J. A., et al. 2004, Proc. SPIE, 5165, 201

Carrasco, L., Porras, A., Recillas, E., et al. 2015, ATel, 6902

Chandra, S., Zhang, H., Kushwaha, P., et al. 2015a, ApJ, 809, 130

Chandra, S., Kushwah, P., Ganesh, S., Kaur, N., \& Baliyan, K. 2015b, ATel, 6962

Chatterjee, R., Jorstad, S. G., \& Marscher, A. P. 2008, ApJ, 689, 79

Chatterjee, R., Bailyn, C. D., Bonning, E. W., et al. 2012, ApJ, 749, 191 
Chen, A. W., D'Ammando, F., Villata, M., et al. 2008, A\&A, 489, L37 Cohen, M. H., Meier, D. L., Arshakian, T. G., et al. 2014, ApJ, 787, 151 Danforth, C. W., Nalewajko, K., France, K., \& Keeney, B. A. 2013, ApJ, 764, 57 Domínguez, A., Primack, J. R., Rosario, D. J., et al. 2011, MNRAS, 410, 2556 Fallah Ramazani, V., Lindfors, E., \& Nilsson, K. 2017, A\&A, 608, A68 Foschini, L., Tagliaferri, G., Pian, E., et al. 2006, A\&A, 455, 871 Franceschini, A., Rodighiero, G., Vaccari, M., et al. 2008, A\&A, 487, 837 Fuhrmann, L., Krichbaum, T. P., Witzel, A., et al. 2008, A\&A, 490, 1019 Fuhrmann, L., Larsson, S., Chiang, J., et al. 2014, MNRAS, 441, 1899 Ghisellini, G., Tavecchio F., \& Chiaberge, M. 2005, A\&A, 432, 401 Giommi, P., Massaro E., Chiappetti, L., et al. 1999, A\&A, 351, 59 Gould R. J., \& Schreder G. P. 1967, Phys. Rev., 155, 1404 Gurwell, M. A., Peck, A. B., Hostler S. R., et al. 2007, ASP Conf. Ser., 375, 234 Harrison, F. A., Craig W. W., Christensen F. E., et al. 2013, ApJ, 770, 103 Hartman, R. C., Bertsch, D. L., Bloom, S. D., et al. 1999, ApJS, 123, 79 Jermak, H., Steele, I. A., Lindfors, E., et al. 2016, MNRAS, 462, 4267 Jorstad, S. G., Marscher, A. P., Mattox, J. R., et al. 2001, ApJ, 556, 738 Jorstad, S. G., Marscher, A. P., Lister, M. L., et al. 2005, AJ, 130, 1418 Jorstad, S. G., Marscher, A. P., Larionov, V. M., et al. 2010, ApJ, 715, 362 Jorstad, S. G., Marscher, A. P., Morozova, D. A, et al. 2017, ApJ, 846, 98 Kalberla, P. M. W., Burton, W. B., Hartmann, D., et al. 2005, A\&A, 440, 775 Larionov, V., Konstantinova, T., Kopatskaya, E., et al. 2008a, ATel, 1502 Larionov, V. M., Jorstad, S. G., Marscher, A. P., et al. 2008b, A\&A, 492, 389 Larionov, V. M., Jorstad, S. G., Marscher, A. P., et al. 2013, ApJ, 768, 40 Li, T.-P., \& Ma,Y.-Q. 1983, ApJ, 272, 317L

Lin, Y. C., Bertsch, D. L., Dingus, B. L., et al. 1995, ApJ, 442, 96 Lister, M. L., Aller, M. F., Aller, H. D., et al. 2013, AJ, 146, 120 Liu, X., Song, H.-G., Marchili, N., et al. 2012, A\&A, 543, A78

Lott, B., Escande, L., Larsson, S., \& Ballet, J. 2012, A\&A, 544, A6

Lyutikov, M., Pariev, V. I., \& Gabuzda, D. C. 2005, MNRAS, 360, 869

Marscher, A. P. 2014, ApJ, 780, 87

Marscher, A. P., Jorstad S. G., D'Arcangelo F. D., et al. 2008, Nature, 452, 966

Marscher, A. P., Jorstad S. G., Larionov, V. M., et al. 2010, ApJ, 710, L126

Mattox, J. R., Bertsch, D. L., Chiang, J., et al. 1996, ApJ, 461, 396

Mirzoyan, R. 2015, ATel, 6999

Mirzoyan, R. 2017, ATel, 11100

Moderski, R., Sikora, M., \& Błażejowski, M. 2003, A\&A, 451, 435

Montagni, F., Maselli, A., Massaro, E., et al. 2006, A\&A, 451, 435

Nilsson, K., Pursimo, T., Sillanpää, A., Takalo, L. O., \& Lindfors, E. 2008, A\&A, 487, L29

Nilsson, K., Lindfors, E., Takalo, L. O., et al. 2018, A\&A, in press, DOI: 10.1051/0004-6361/201833621

Paiano, S., Landoni, M., Falomo, R., et al. 2017, ApJ, 837, 144

Poole, T. S., Breeveld, A. A., Page, M. J., et al. 2008, MNRAS, 383, 627

Poutanen, J., Zdziarski, A. A., \& Ibragimov, A. 2008, MNRAS, 389, 1427

Prandini, E., Bonnoli, G., Maraschi, L., Mariotti, M., \& Tavecchio, F. 2010, MNRAS, 405, L76

Prandini, E., Bonnoli, G., Maraschi, L., Mariotti, M., \& Tavecchio, F. 2011, PoS(CRF 2010)012

Quirrenbach, A., Witzel, A., Wagner, S., et al. 1991, ApJ, 372, L71

Raiteri, C. M., Villata, M., Tosti, G., et al. 2003, A\&A, 402, 151

Rani, B., Gupta, A. C., Joshi, U. C., Ganesh, S., \& Wiita, P. J. 2010, ApJ, 719, L153

Rani, B., Gupta, A. C., Joshi, U. C., Ganesh, S., \& Wiita, P. J. 2011, MNRAS, 413, 2157

Rani, B., Krichbaum, T. P., Fuhrmann, L., et al. 2013, A\&A, 552, A11

Rani, B., Krichbaum, T. P., Marscher, A. P., et al. 2014, A\&A, 571, L2

Rani, B., Krichbaum, T. P., Marscher, A. P., et al. 2015, A\&A, 578, A123

Rastorgueva, E. A., Wiik, K. J., Bajkova, A. T., et al. 2011, A\&A, 529, A2

Richards, J. L., Max-Moerbeck, W., Pavlidou, V., et al. 2011, ApJS, 194, 29

Sault, R. J., Teuben, P. J., \& Wright, M. C. H. 1995, ASP Conf. Ser., 77, 436

Schlafly, E. F., \& Finkbeiner, D. P. 2011, ApJ, 737, 103

Schulz, A., \& Lenzen, R. 1983, A\&A, 121, 158

Senturk, G. D., Errando, M., Boettcher, M., et al. 2013, ApJ, 764, 119

Spiridonova, O. I., Vlasyuk, V. V., Moskvitin, A. S., \& Bychkova, V. S. 2015, ATel, 7004

Stecker, F. W., DeJager, O. C., \& Salamon, M. H. 1992, ApJ, 390, L49

Steele, I. A., Kopač, D., Arnold, D. M., et al. 2017, ApJ, 843, 143

Tagliaferri, G., Ravasio, M., Ghisellini, G., et al. 2003, A\&A, 400, 477

Tavecchio, F., \& Ghisellini, G. 2009, MNRAS, 394, 13

Tavecchio, F., Becerra-Gonzalez, J., Ghisellini, G., et al. 2011, A\&A, 534, A86

Teräsranta, H., Tornikoski, M., Mujunen, A., et al. 1998, A\&AS, 132, 305

Thum, C., Agudo, I., Molina, S. N., et al. 2018, MNRAS, 473, 2506

Uttley, P., Edelson, R., McHardy, I. M., et al. 2003 ApJ, 584, L53

Vaughan, S., Edelson, R., Warwick, R. S., \& Uttley, P. 2003, MNRAS, 345, 1271

Wagner, S. J., Witzel, A., Heidt, J., et al. 1996, AJ, 111, 2187

Wierzcholska, A., \& Siejkowski, H. 2016, MNRAS, 458, 2350

Witzel, A., Schalinski, C. J., Johnston, K. J., et al. 1988, A\&A, 206, 245
Zanin, R., Carmona, E., Sitarek, J., et al. 2013, Proc. 33th International Cosmic Ray Conference (ICRC), 773

Zhang, H., Chen, X., \& Böttcher, M. 2014, ApJ, 789, 66

${ }^{1}$ ETH Zurich, 8093 Zurich, Switzerland

2 Università di Udine; INFN Trieste, 33100 Udine, Italy

3 National Institute for Astrophysics (INAF), 00136 Rome, Italy

${ }^{4}$ Università di Padova, Italy

5 Technische Universität Dortmund, 44221 Dortmund, Germany

${ }^{6}$ Croatian MAGIC Consortium: University of Rijeka, 51000 Rijeka; University of Split - FESB, 21000 Split; University of Zagreb FER, 10000 Zagreb; University of Osijek, 31000 Osijek; Rudjer Boskovic Institute, 10000 Zagreb, Croatia

7 Saha Institute of Nuclear Physics, HBNI, 1/AF Bidhannagar, Salt Lake, Sector-1, Kolkata 700064, India

8 Max-Planck-Institut für Physik, 80805 München, Germany

9 Centro Brasileiro de Pesquisas Físicas (CBPF), 22290-180 URCA, Rio de Janeiro (RJ), Brasil

10 Unidad de Partículas y Cosmología (UPARCOS), Universidad Complutense, 28040 Madrid, Spain

11 Inst. de Astrofísica de Canarias, 38200 La Laguna, Universidad de La Laguna, Dpto. Astrofísica, 38206 La Laguna, Tenerife, Spain

12 University of Łódź, Department of Astrophysics, 90236 Łódź, Poland

13 Deutsches Elektronen-Synchrotron (DESY), 15738 Zeuthen, Germany

14 Institut de Física d'Altes Energies (IFAE), The Barcelona Institute of Science and Technology (BIST), 08193 Bellaterra (Barcelona), Spain

15 Università di Siena and INFN Pisa, 53100 Siena, Italy

16 Universität Würzburg, 97074 Würzburg, Germany

17 Finnish MAGIC Consortium: Tuorla Observatory (Department of Physics and Astronomy) and Finnish Centre of Astronomy with ESO (FINCA), University of Turku, 20014 Turku, Finland; Astronomy Division, University of Oulu, 90014 Oulu, Finland

18 Departament de Física; CERES-IEEC, Universitat Autónoma de Barcelona, 08193 Bellaterra, Spain

19 Universitat de Barcelona, ICC, IEEC-UB, 08028 Barcelona, Spain

20 Japanese MAGIC Consortium: ICRR, The University of Tokyo, 277-8582 Chiba, Japan; Department of Physics, Kyoto University, 606-8502 Kyoto, Japan; Tokai University, 259-1292 Kanagawa, Japan; The University of Tokushima, 770-8502 Tokushima, Japan

21 Inst. for Nucl. Research and Nucl. Energy, Bulgarian Academy of Sciences, 1784 Sofia, Bulgaria

22 Università di Pisa and INFN Pisa, 56126 Pisa, Italy

23 Humboldt University of Berlin, Institut für Physik, 12489 Berlin, Germany

24 Dipartimento di Fisica, Università di Trieste, 34127 Trieste, Italy

25 Port d'Informació Científica (PIC), 08193 Bellaterra (Barcelona), Spain

26 INAF-Trieste, Dept. of Physics \& Astronomy, University of Bologna, Italy

27 INFN, 35131 Padova, Italy

28 ASI Science Data Center and INFN, 06123 Perugia, Italy

${ }^{29}$ CEN Bordeaux-Gradignan, 33170 Gradignan, France

30 NASA Goddard Space Flight Center, Greenbelt, MD 20771, USA

31 Instituto de Astrofísica de Andalucía (CSIC), Apartado 3004, 18080 Granada, Spain

32 Max-Planck-Institut für Radioastronomie, Auf dem Hügel, 69, 53121 Bonn, Germany

33 Crimean Astrophysics Observatory, P/O Nauchny 298409, Crimea

34 Astron. Inst., St. Petersburg State University, Russia

35 Harvard-Smithsonian Center for Astrophysics, MA, 02138 Cambridge, USA

36 Metsähovi Radio Observatory, Aalto University, 02540 Kylmälä, Finland

37 Department of Electronics and Nanoengineering, Aalto University, 00076 Aalto, Finland

38 Tuorla Observatory, University of Turku, Väisäläntie 20, 21500 Piikkiö, Finland 
39 Department of Physical Science, Hiroshima University, Higashihiroshima 739-8526, Japan

40 Institute for Astrophysical Research, Boston University, Boston, MA 02215, USA

41 Mullard Space Science Lab., UCL, Dorking RH5 6NT, UK

${ }_{42}$ Pulkovo Observatory, St.-Petersburg, Russia

43 Kavli Institute for Particle Astrophysics and Cosmology, Stanford University and SLAC National Accelerator Laboratory, 2575 Sand Hill Road, Menlo Park, CA 94025, USA
44 Nicolaus Copernicus Astronomical Center, Polish Academy of Sciences, Bartycka 18, 00-716 Warsaw, Poland

45 Owens Valley Radio Observatory, California Institute of Technology, Pasadena, CA 91125, USA

46 CePIA, Astronomy Department, Universidad de Concepcion, Casilla 160-C, Concepcion, Chile

47 Astrophysics Research Institute, Liverpool John Moores University, Brownlow Hill, Liverpool L3 5RF, UK 
M. L. Ahnen et al.: MWL characterization of S5 0716+714 during an unprecedented outburst

Appendix A: Knot parameters for S5 0716+714 (July 2014-August 2015)

In Table A. 1 the parameters of components from Sect. 4 are listed; they indicate the flux density, $S$, the relative RA and Dec with respect to the core, the distance from the core $r$, the position angle with respect to the core, $\Theta$, and the size of the component, $a$ (FWHM of the Gaussian) for every knot. Some knots present in Table A.1 do not have any assigned name because they have been seen at 1-2 epochs only.

Table A.1. Knot parameters for S5 0716+714 (July 2014-August 2015).

\begin{tabular}{|c|c|c|c|c|c|c|c|c|}
\hline Epoch & MJD & $S(\mathrm{Jy})$ & $x$ (mas) & $y$ (mas) & $r$ (mas) & $\Theta(\operatorname{deg})$ & $a$ (mas) & Knot \\
\hline \multicolumn{9}{|c|}{ - 2014-Jul-28 - } \\
\hline 2014.5726 & 56867 & 1.619 & 0.000 & 0.000 & 0.000 & 0.0 & 0.020 & A0 \\
\hline 2014.5726 & 56867 & 0.548 & 0.092 & 0.005 & 0.093 & 86.8 & 0.031 & A1 \\
\hline 2014.5726 & 56867 & 0.017 & 0.186 & 0.551 & 0.582 & 18.7 & 0.186 & \\
\hline \multicolumn{9}{|c|}{ - 2014-Sep-23 - } \\
\hline 2014.7288 & 56924 & 1.322 & 0.000 & 0.000 & 0.000 & 0.0 & 0.020 & $\mathrm{~A} 0$ \\
\hline 2014.7288 & 56924 & 0.205 & 0.095 & 0.021 & 0.097 & 77.6 & 0.031 & A1 \\
\hline 2014.7288 & 56924 & 0.033 & 0.158 & 0.457 & 0.484 & 19.1 & 0.189 & \\
\hline \multicolumn{9}{|c|}{ - 2014-Nov-15 - } \\
\hline 2014.8740 & 56977 & 2.676 & 0.000 & 0.000 & 0.000 & 0.0 & 0.022 & A0 \\
\hline 2014.8748 & 56977 & 0.105 & 0.095 & 0.021 & 0.097 & 77.6 & 0.031 & A1 \\
\hline 2014.8740 & 56977 & 0.306 & 0.178 & 0.095 & 0.202 & 62.0 & 0.112 & $\mathrm{~K} 14 \mathrm{a}$ \\
\hline \multicolumn{9}{|c|}{ - 2014-Dec-5 - } \\
\hline 2014.9288 & 56997 & 1.717 & 0.000 & 0.000 & 0.000 & 0.0 & 0.019 & A0 \\
\hline 2014.9288 & 56997 & 0.571 & 0.078 & 0.055 & 0.095 & 54.8 & 0.038 & A1 \\
\hline 2014.9288 & 56997 & 0.183 & 0.206 & 0.139 & 0.248 & 56.1 & 0.094 & $\mathrm{~K} 14 \mathrm{a}$ \\
\hline 2014.9288 & 56997 & 0.019 & 0.163 & 0.440 & 0.469 & 20.3 & 0.274 & \\
\hline \multicolumn{9}{|c|}{ - 2014-Dec-29 - } \\
\hline 2014.9945 & 57021 & 1.171 & 0.000 & 0.000 & 0.000 & 0.0 & 0.017 & A0 \\
\hline 2014.9945 & 57021 & 0.168 & 0.093 & 0.077 & 0.121 & 50.5 & 0.023 & A1 \\
\hline 2014.9945 & 57021 & 0.245 & 0.250 & 0.179 & 0.307 & 54.4 & 0.130 & $\mathrm{~K} 14 \mathrm{a}$ \\
\hline 2014.9945 & 57021 & 0.021 & 0.246 & 0.602 & 0.651 & 22.2 & 0.416 & \\
\hline \multicolumn{9}{|c|}{ - 2015-Feb14 - } \\
\hline 2015.1233 & 57067 & 1.901 & 0.000 & 0.000 & 0.000 & 0.0 & 0.013 & A0 \\
\hline 2015.1233 & 57067 & 0.314 & 0.093 & 0.080 & 0.123 & 49.2 & 0.065 & A1 \\
\hline 2015.1233 & 57067 & 0.122 & 0.232 & 0.222 & 0.321 & 46.3 & 0.155 & $\mathrm{~K} 14 \mathrm{a}$ \\
\hline 2015.1233 & 57067 & 0.036 & 0.236 & 0.527 & 0.577 & 24.1 & 0.412 & \\
\hline \multicolumn{9}{|c|}{ - 2015-Apr-11 - } \\
\hline 2015.2767 & 57123 & 2.094 & 0.000 & 0.000 & 0.000 & 0.0 & 0.017 & $\mathrm{~A} 0$ \\
\hline 2015.2767 & 57123 & 0.373 & 0.069 & 0.056 & 0.089 & 51.3 & 0.057 & A1 \\
\hline 2015.2767 & 57123 & 0.045 & 0.163 & 0.169 & 0.235 & 44.1 & 0.100 & $\mathrm{~K} 14 \mathrm{~b}$ \\
\hline 2015.2767 & 57123 & 0.089 & 0.287 & 0.390 & 0.484 & 36.3 & 0.179 & $\mathrm{~K} 14 \mathrm{a}$ \\
\hline \multicolumn{9}{|c|}{ - 2015-May-11 - } \\
\hline 2015.3589 & 57153 & 1.581 & 0.000 & 0.000 & 0.000 & 0.0 & 0.023 & A0 \\
\hline 2015.3589 & 57153 & 0.309 & 0.074 & 0.065 & 0.098 & 48.7 & 0.063 & A1 \\
\hline 2015.3589 & 57153 & 0.032 & 0.204 & 0.165 & 0.262 & 50.9 & 0.095 & $\mathrm{~K} 14 \mathrm{~b}$ \\
\hline 2015.3589 & 57153 & 0.069 & 0.286 & 0.407 & 0.498 & 35.1 & 0.201 & $\mathrm{~K} 14 \mathrm{a}$ \\
\hline \multicolumn{9}{|c|}{ - 2015-Jun-9 - } \\
\hline 2015.4385 & 57182 & 0.947 & 0.000 & 0.000 & 0.000 & 0.0 & 0.018 & A0 \\
\hline 2015.4385 & 57182 & 0.308 & 0.106 & 0.098 & 0.144 & 47.5 & 0.067 & A1 \\
\hline 2015.4385 & 57182 & 0.039 & 0.229 & 0.191 & 0.298 & 50.3 & 0.069 & $\mathrm{~K} 14 \mathrm{~b}$ \\
\hline 2015.4385 & 57182 & 0.054 & 0.320 & 0.437 & 0.542 & 36.2 & 0.189 & $\mathrm{~K} 14 \mathrm{a}$ \\
\hline
\end{tabular}


A\&A 619, A45 (2018)

Table A.1. continued.

\begin{tabular}{ccccccccc}
\hline \hline & \multicolumn{7}{c}{ - 2015-Jul-2- } \\
\hline 2015.5014 & 57205 & 1.612 & 0.000 & 0.000 & 0.000 & 0.0 & 0.021 & A0 \\
2015.5014 & 57205 & 0.124 & 0.020 & 0.060 & 0.063 & 18.7 & 0.000 & K15 \\
2015.5014 & 57205 & 0.144 & 0.075 & 0.096 & 0.122 & 37.9 & 0.084 & A1 \\
2015.5014 & 57205 & 0.041 & 0.253 & 0.204 & 0.325 & 51.1 & 0.084 & K14b \\
2015.5014 & 57205 & 0.057 & 0.322 & 0.384 & 0.531 & 40.0 & 0.141 & K14a \\
2015.5014 & 57205 & 0.016 & 0.376 & 0.665 & 0.764 & 29.5 & 0.401 & \\
\hline & & & $-2015-$ Aug-1 - & & & \\
\hline 2015.5836 & 57235 & 1.865 & 0.000 & 0.000 & 0.000 & 0.0 & 0.019 & A0 \\
2015.5836 & 57235 & 0.238 & 0.045 & 0.090 & 0.101 & 26.3 & 0.033 & K15 \\
2015.5836 & 57235 & 0.245 & 0.087 & 0.126 & 0.153 & 34.6 & 0.083 & A1 \\
2015.5836 & 57235 & 0.032 & 0.241 & 0.278 & 0.368 & 41.0 & 0.107 & K14b \\
2015.5836 & 57235 & 0.018 & 0.388 & 0.455 & 0.598 & 40.4 & 0.119 & K14a \\
2015.5836 & 57235 & 0.020 & 0.315 & 0.667 & 0.738 & 25.3 & 0.398 & \\
\hline
\end{tabular}

